\title{
Philosophy of Science for Scientists: The Probabilistic Interpretation of Science
}

\author{
Shiro Ishikawa \\ Department of Mathematics, Faculty of Science and Technology, Keio University, Yokohama, Japan \\ Email: ishikawa@math.keio.ac.jp
}

How to cite this paper: Ishikawa, S. (2019) Philosophy of Science for Scientists: The Probabilistic Interpretation of Science. Journal of Quantum Information Science, 9, 123-154.

https://doi.org/10.4236/jqis.2019.93007

Received: August 27, 2019

Accepted: September 24, 2019

Published: September 27, 2019

Copyright ( 2019 by author(s) and Scientific Research Publishing Inc. This work is licensed under the Creative Commons Attribution International License (CC BY 4.0).

http://creativecommons.org/licenses/by/4.0/

(c) (i) Open Access

\begin{abstract}
Recently we proposed "quantum language" (or, "the linguistic Copenhagen interpretation of quantum mechanics", "measurement theory") as the language of science. This theory asserts the probabilistic interpretation of science (=the linguistic quantum mechanical worldview), which is a kind of mathematical generalization of Born's probabilistic interpretation of quantum mechanics. In this paper, we consider the most fundamental problems in philosophy of science such as Hempel's raven paradox, Hume's problem of induction, Goodman's grue paradox, Peirce's abduction, flagpole problem, which are closely related to measurement. We believe that these problems can never be solved without the basic theory of science with axioms. Since our worldview (=quantum language) has the axiom concerning measurement, these problems can be solved easily. Thus we believe that quantum language is the central theory in philosophy of science. Hence there is a reason to assert that quantum language gives the mathematical foundations to science.
\end{abstract}

\section{Keywords}

Philosophy of Science, Linguistic Copenhagen Interpretation, Probabilistic Interpretation of Science, Raven Paradox, Problem of Induction, Grue Paradox, Abduction, Flagpole Problem

\section{Introduction}

\subsection{Philosophy of Science Is about as Useful to Scientists as Ornithology Is to Birds}

We think that philosophy of science is classified as follows.

$\left(A_{1}\right)$ Criticism about science, The relation between society and science, History of science, etc.

(Non-scientists may be interested in these mainly, thus it is called "philosophy 
of science for the general public" in this paper)

$\left(A_{2}\right)$ Study on mathematical foundations of science, i.e., research on mathematical properties common to various sciences, that is, a kind of unified theory of science

(This is called "philosophy of science for scientists" in this paper)

The part $\left(A_{1}\right)$ is a large part of the philosophy of science. And it is certain that many valuable results have been produced in the study $\left(A_{1}\right)$ (e.g., Popper's falsificationism ( $c f$. Remark 18 later)). On the other hand, it is generally considered that the study $\left(\mathrm{A}_{2}\right)$ was not so productive in spite that the part $\left(\mathrm{A}_{2}\right)$ (e.g., Hempel's scientific explanation ( $c f$. refs. [1] [2])) was expected to be the core of philosophy of science. For example, Prof. Richard Feynman, one of the most outstanding physicists in the 20th century, said that "Philosophy of science is about as useful to scientists as ornithology is to birds". If he used the word "philosophy of science" to mean "philosophy of science for scientists", we can agree with his claim. That is, we also consider that the part $\left(A_{2}\right)$ is still undeveloped. Thus, our purpose of this paper is to complete "philosophy of science for scientists" in $\left(A_{2}\right)$.

This will be done by using quantum language (mentioned in Section 2), which has two fundamental concepts "measurement" and "causality" (that are common to various sciences). And in Sections 3-6, we show that famous unsolved problems (i.e., Hempel's raven paradox, Hume's problem of induction, Goodman's grue paradox, Peirce's abduction, flagpole problem) in philosophy of science can be solved in quantum language.

\section{Review: Quantum Language (=Measurement Theory (=MT))}

\subsection{Quantum Language Is the Language to Describe Science}

Recently, in refs. [3]-[18], we proposed quantum language (or, "the linguistic Copenhagen interpretation of quantum mechanics", "measurement theory $(=\mathrm{MT})$ "), which is a kind of the language of science. This is not only characterized as the metaphysical and linguistic turn of quantum mechanics but also the quantitative turn of Descartes = Kant epistemology and the dualistic turn of statistics. Thus, the location of this theory in the history of scientific worldviews is as follows (cf. refs. [8] [14] [18]):

Figure 1 says that quantum language (=the linguistic Copenhagen interpretation) has the following three aspects

$\left(B_{1}\right)$ the linguistic Copenhagen interpretation is the true figure of so-called Copenhagen interpretation (7) in Figure 1), cf. refs. [3] [6] [7] [13] [16], particularly, Heisenberg's uncertainty relation was proved in [3], and the von Neumann-Lüders projection postulate (i.e., the meaning of the wave function collapse) was clarified in [13],

$\left(B_{2}\right)$ the scientific final goal of dualistic idealism (i.e., Descartes = Kant philosophy) (8) in Figure 1), cf. refs. [8] [14] [15] [17], particularly, the mind-body problem was clarified in [15], also, the paradox of brain in bat (cf. [19]) was 
solved in [17],

$\left(\mathrm{B}_{3}\right)$ statistics (=dynamical system theory) with the concept measurement (9) in Figure 1), cf. refs. [4] [5] [6] [10] [11]. Thus, quantum language gives the answer of "Why statistics is used in science?"

Hence, it is natural to assume that

$\left(B_{4}\right)$ quantum language proposes the probabilistic interpretation of science (=the linguistic quantum mechanical worldview), and thus, it is just the language to describe science. Thus, we assert that science is built on dualism ( $\approx$ ["observer" + "matter"] $\approx$ measurement) and idealism ( $\approx$ metaphysics $\approx$ language).

which is the most important assertion of quantum language. Also, we assume that to make the language to describe science is one of main purposes of philosophy of science.

As criticism of philosophy of science, there is criticism that scientific philosophy is not very useful for scientists (as mentioned in Section 1). We agree to this criticism. However, as mentioned in the above $\left(B_{1}\right)$ and $\left(B_{3}\right)$, we say that quantum language is one of the most useful theories in science, and thus it should be regarded as a kind of unified theory of science.

Remark 1. Since space and time are independent in quantum language, the theory of relativity (and further, the theory of everything: (5) in Figure 1) cannot be described by quantum language. We think that the theory of relativity is too special, an exception. It is too optimistic to expect that all scientific propositions can be written in quantum language. However, we want to assert the $\left(\mathrm{B}_{4}\right)$, that is, quantum language is the most fundamental language for almost all familiar science. We believe that arguments without a worldview do not bring about the success of philosophy of science.

\subsection{No Scientific Argument without Scientific Worldview}

It is well known that the following problems are the most fundamental in philosophy of science:

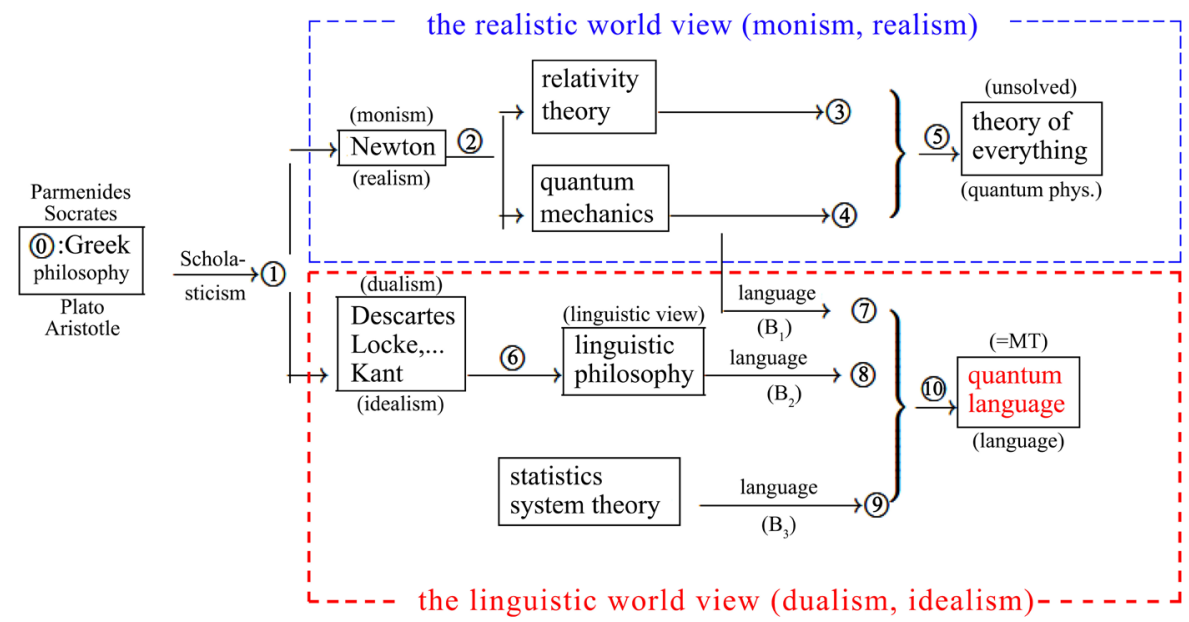

Figure 1. The location of quantum language in the history of the world-description. 
(C) Hempel's raven paradox, Goodman's grue paradox, Hume's problem of induction, Peirce's abduction, the flagpole problem etc.

In Sections 3-6, we clarify these problems under the linguistic quantum mechanical worldview $\left(\mathrm{B}_{4}\right)$, since we believe that there is no scientific argument without scientific worldview (or, without scientific language). That is, the above are not problems in mathematics and logic. And we conclude that the reason that these problems are not yet clarified depends on lack of the concept of measurement in philosophy of science.

\subsection{Mathematical Preparations}

Following refs. [6] [7] [8] [18] (all our results until present are written in ref. [18]), we shall review quantum language, which has the following form:

$$
\begin{aligned}
& \frac{\text { Quantumlanguage }}{\text { (=measurement theory) }} \\
& =\underset{\text { (Axiom 1) }}{\text { measurement }}+\underset{\text { (Axiom 2) }}{\text { causality }}+\frac{\text { linguistic(Copenhagen) interpretation }}{\text { (how to use Axioms 1 and 2) }}
\end{aligned}
$$

which asserts that "measurement" and "causality" are the most important concepts in science.

Consider an operator algebra $B(H)$ (i.e., an operator algebra composed of all bounded linear operators on a Hilbert space $H$ with the norm $\|F\|_{B(H)}=\sup _{\|u\|_{H}=1}\|F u\|_{H}$ ), and consider the triplet $[\mathcal{A} \subseteq \mathcal{N} \subseteq B(H)]$ (or, the pair $\left.[\mathcal{A}, \mathcal{N}]_{B(H)}\right)$, called a basic structure. Here, $\mathcal{A}(\subseteq B(H))$ is a $C$-algebra, and $\mathcal{N}(\mathcal{A} \subseteq \mathcal{N} \subseteq B(H))$ is a particular $C^{*}$-algebra (called a $W^{*}$-algebra) such that $\mathcal{N}$ is the weak closure of $\mathcal{A}$ in $B(H)$.

The measurement theory (="quantum language" = "the linguistic Copenhagen interpretation") is classified as follows.

(D) $\underset{\text { (=quantum language) }}{\operatorname{measurement}}=\left\{\begin{array}{l}\left(\mathrm{D}_{1}\right): \text { quantum system theory }(\text { when } \mathcal{A}=\mathcal{C}(H)) \\ \left(\mathrm{D}_{2}\right) \text { : classical system theory }\left(\text { when } \mathcal{A}=C_{0}(\Omega)\right)\end{array}\right.$

The Hilbert space method for the mathematical foundations of quantum mechanics is essentially due to von Neumann (cf. ref. [20]). He devoted himself to quantum $\left(D_{1}\right)$. On the other hand, in most cases, we devote ourselves to classical $\left(D_{2}\right)$, and not $\left(D_{1}\right)$. However, the quantum $\left(D_{1}\right)$ is convenient for us, in the sense that the idea in $\left(\mathrm{D}_{1}\right)$ is often introduced into classical $\left(\mathrm{D}_{2}\right)$.

When $\mathcal{A}=\mathcal{C}(H)$, the $C$-algebra composed of all compact operators on a Hilbert space $H$, the $\left(\mathrm{D}_{1}\right)$ is called quantum measurement theory (or, quantum system theory), which can be regarded as the linguistic aspect of quantum mechanics. Also, when $\mathcal{A}$ is commutative (that is, when $\mathcal{A}$ is characterized by $C_{0}(\Omega)$, the $C$-algebra composed of all continuous complex-valued functions vanishing at infinity on a locally compact Hausdorff space $\Omega$ (cf. refs. [21] [22] [23])), the $\left(D_{2}\right)$ is called classical measurement theory.

Also, note that, when $\mathcal{A}=\mathcal{C}(H)$, i.e., quantum cases, 
(E $\left.\mathrm{E}_{1}\right) \mathcal{A}^{*}\left(=\mathcal{C}(H)^{*}\right)=\operatorname{Tr}(H) \quad$ (=trace class), $\mathcal{N}=B(H), \mathcal{N}_{*}=\operatorname{Tr}(H) \quad$ i.e., pre-dual space), thus, $\operatorname{Tr}(H)(\rho, T)_{B(H)}=\operatorname{Tr}_{H}(\rho T) \quad(\rho \in \operatorname{Tr}(H), T \in B(H))$.

Also, when $\mathcal{A}=C_{0}(\Omega)$, i.e., classical cases,

(E $\left.\mathrm{E}_{2}\right) \mathcal{A}^{*}\left(=C_{0}(\Omega)^{*}\right)=\mathcal{M}(\Omega)$ i.e., "the space of all signed measures on $\Omega$ ", $\mathcal{N}=L^{\infty}(\Omega, v)\left(\subseteq B\left(L^{2}(\Omega, v)\right)\right), \quad \mathcal{N}_{*}=L^{1}(\Omega, v)$, where $v$ is some measure on $\Omega$ (with the Borel field $\mathcal{B}$, thus, ${ }_{L^{1}(\Omega, v)}(\rho, T)_{L^{\infty}(\Omega, v)}=\int_{\Omega} \rho(\omega) T(\omega) v(\mathrm{~d} \omega)$ $\left(\rho \in L^{1}(\Omega, v), T \in L^{\infty}(\Omega, v)\right.$ ) (cf. ref. [22]).

In Sections 3-6 later, we devote ourselves to a compact space $\Omega$ with a probability measure $v$ (i.e., $v(\Omega)=1$ ) and thus, $C_{0}(\Omega)$ is simply denoted by $C(\Omega)$.

Let $\mathcal{A}(\subseteq B(H))$ be a $C$-algebra, and let $\mathcal{A}^{*}$ be the dual Banach space of $\mathcal{A}$. That is, $\mathcal{A}^{*}=\{\rho \mid \rho$ is a continuous linear functional on $\mathcal{A}\}$, and the norm $\|\rho\|_{\mathcal{A}^{*}}$ is defined by $\sup \left\{|\rho(F)| \mid F \in \mathcal{A}\right.$ such that $\left.\|F\|_{\mathcal{A}}\left(=\|F\|_{B(H)}\right) \leq 1\right\}$. Define the mixed state $\rho\left(\in \mathcal{A}^{*}\right)$ such that $\|\rho\|_{\mathcal{A}^{*}}=1$ and $\rho(F) \geq 0$ for all $F \in \mathcal{A}$ such that $F \geq 0$. And define the mixed state space $\mathfrak{S}^{m}\left(\mathcal{A}^{*}\right)$ such that

$$
\mathfrak{S}^{m}\left(\mathcal{A}^{*}\right)=\left\{\rho \in \mathcal{A}^{*} \mid \rho \text { is a mixed state }\right\} .
$$

A mixed state $\rho\left(\in \mathfrak{S}^{m}\left(\mathcal{A}^{*}\right)\right)$ is called a pure state if it satisfies that $\rho=\theta \rho_{1}+(1-\theta) \rho_{2} \quad$ for some $\rho_{1}, \rho_{2} \in \mathfrak{S}^{m}\left(\mathcal{A}^{*}\right)$ and $0<\theta<1 \quad$ implies $\rho=\rho_{1}=\rho_{2}$. Put

$$
\mathfrak{S}^{p}\left(\mathcal{A}^{*}\right)=\left\{\rho \in \mathfrak{S}^{m}\left(\mathcal{A}^{*}\right) \mid \rho \text { is a pure state }\right\},
$$

which is called a state space. It is well known (cf. ref. [22]) that $\mathfrak{S}^{p}\left(\mathcal{C}(H)^{*}\right)=\left\{|u\rangle\langle u|(\right.$ i.e., the Dirac notation $\left.) \mid\|u\|_{H}=1\right\}$, and $\mathfrak{S}^{p}\left(C_{0}(\Omega)^{*}\right)=\left\{\delta_{\omega_{0}} \mid \delta_{\omega_{0}}\right.$ is a point measure at $\left.\omega_{0} \in \Omega\right\}$, where $\int_{\Omega} f(\omega) \delta_{\omega_{0}}(\mathrm{~d} \omega)=f\left(\omega_{0}\right) \quad\left(\forall f \in C_{0}(\Omega)\right)$. The latter implies that $\mathfrak{S}^{p}\left(C_{0}(\Omega)^{*}\right)$ can be also identified with $\Omega$ (called a spectrum space or simply spectrum) such as

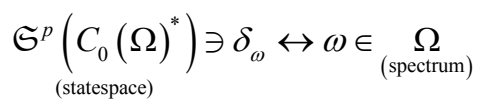

In this paper, $\Omega$ and $\omega(\in \Omega)$ is respectively called a state space and a state. In Axiom 1 later, we need the value of ${ }_{\mathcal{A}^{*}}(\rho, G)_{\mathcal{N}}$, where $\rho \in \mathfrak{S}^{p}\left(\mathcal{A}^{*}\right)$, $G \in \mathcal{N}$. In quantum cases, we see that $\mathcal{A}^{*}(\rho, G)_{\mathcal{N}}={ }_{\operatorname{Tr}(H)}(\rho, G)_{B(H)}$. Thus, the value of $\mathcal{A}^{*}(\rho, G)_{\mathcal{N}}$ is clearly determined. However, in classical cases (i.e., $\left.\mathcal{A}^{*}(\rho, G)_{\mathcal{N}}={ }_{\mathcal{M}(\Omega)}(\rho, G)_{L^{\infty}(\Omega, v)}\right)$, we have to prepare the following definition.

Definition 2. [Essentially continuous in classical cases] An element $F\left(\in L^{\infty}(\Omega, v)\right)$ is said to be essentially continuous at $\delta_{\omega_{0}}\left(\in \mathfrak{S}^{p}(\mathcal{M}(\Omega))\right)$ (i.e., 
at $\left.\omega_{0}(\in \Omega)\right)$, if there uniquely exists a complex number $\alpha$ such that

- if $\rho\left(\in L^{1}(\Omega, v),\|\rho\|_{L^{1}(\Omega, v)}=1, \rho \geq 0\right)$ converges to $\delta_{\omega_{0}}\left(\in \mathfrak{S}^{p}(\mathcal{M}(\Omega))\right)$ in the sense of weak topology of $\mathcal{M}(\Omega)$, that is,

$$
\begin{aligned}
& \rho(G)\left(=\int_{\Omega} G(\omega) \rho(\omega) v(\mathrm{~d} \omega)\right) \\
& \rightarrow \int_{\Omega} G(\omega) \delta_{\omega_{0}}(\mathrm{~d} \omega)\left(=G\left(\omega_{0}\right)\right) \quad\left(\forall G \in C_{0}(\Omega)\left(\subseteq L^{\infty}(\Omega, v)\right)\right),
\end{aligned}
$$

then $\rho(F)$ converges to $\alpha$.

The following definition is due to E.B. Davies (cf. ref. [24]).

Definition 3. [Observable] An observable $\mathrm{O}=(X, \mathcal{F}, F)$ in $\mathcal{N}$ is defined as follows:

1) [ $\sigma$-field] $X$ is a set, $\mathcal{F}\left(\subseteq 2^{X} \equiv \mathcal{P}(X)\right.$, the power set of $\left.X\right)$ is a $\sigma$-field of $X$, that is, " $\Xi_{1}, \Xi_{2}, \cdots \in \mathcal{F} \Rightarrow \bigcup_{n=1}^{\infty} \Xi_{n} \in \mathcal{F}$ ”, “ $\Xi \in \mathcal{F} \Rightarrow X \backslash \Xi\left(\equiv\{x \mid x \in X, x \notin \Xi\} \equiv \Xi^{c}\right.$, i.e., the complement of $\left.\Xi\right) \in \mathcal{F}$ ”.

2) [Countable additivity] $F$ is a mapping from $\mathcal{F}$ to $\mathcal{N}$ satisfying: a): for every $\Xi \in \mathcal{F}, \quad F(\Xi)$ is a non-negative element in $\mathcal{N}$ such that $0 \leq F(\Xi) \leq I, \mathrm{~b}): F(\varnothing)=0$ and $F(X)=I$, where 0 and $I$ is the 0 -element and the identity in $\mathcal{N}$ respectively. c): for any countable decomposition $\left\{\Xi_{1}, \Xi_{2}, \cdots, \Xi_{n}, \cdots\right\} \quad$ of $\Xi \quad$ (i.e., $\quad \Xi, \Xi_{n} \in \mathcal{F}(n=1,2,3, \cdots), \quad \bigcup_{n=1}^{\infty} \Xi_{n}=\Xi$, $\left.\Xi_{i} \cap \Xi_{j}=\varnothing \quad(i \neq j)\right)$, it holds that $F(\Xi)=\sum_{n=1}^{\infty} F\left(\Xi_{n}\right)$ in the sense of weak topology in $\mathcal{N}$.

\subsection{Axiom 1 [Measurement] and Axiom 2 [Causality]}

Quantum language (1) is composed of two axioms (i.e., Axioms 1 and 2) as follows. With any system $S$, a basic structure $[\mathcal{A} \subseteq \mathcal{N} \subseteq B(H)]$ can be associated in which the measurement theory (A) of that system can be formulated. A state of the system $S$ is represented by an element $\rho\left(\in \mathfrak{S}^{p}\left(\mathcal{A}^{*}\right)\right)$ and an observable is represented by an observable $\mathrm{O}=(X, \mathcal{F}, F)$ in $\mathcal{N}$. Also, the measurement of the observable $\mathrm{O}$ for the system $S$ with the state $\rho$ is denoted by $\mathrm{M}_{\mathcal{N}}\left(\mathrm{O}, S_{[\rho]}\right)$ (or more precisely, $\mathrm{M}_{\mathcal{N}}\left(\mathrm{O}:=(X, \mathcal{F}, F), S_{[\rho]}\right)$ ). An observer can obtain a measured value $x(\in X)$ by the measurement $\mathrm{M}_{\mathcal{N}}\left(\mathrm{O}, S_{[\rho]}\right)$.

The Axiom 1 presented below is a kind of mathematical generalization of Born's probabilistic interpretation of quantum mechanics. And thus, it is a statement without reality.

Now we can present Axiom 1 in the $W^{*}$-algebraic formulation as follows.

Axiom 1 [Measurement, the probabilistic interpretation of science]. The probability that a measured value $x(\in X)$ obtained by the measurement $\mathrm{M}_{\mathcal{N}}\left(\mathrm{O}:=(X, \mathcal{F}, F), S_{[\rho]}\right)$ belongs to a set $\Xi(\in \mathcal{F})$ is given by $\rho(F(\Xi))$ if $F(\Xi)$ is essentially continuous at $\rho\left(\in \mathfrak{S}^{p}\left(\mathcal{A}^{*}\right)\right)$.

This axiom gives answers to "What is probability?" and "What is measurement?".

Remark 4. In quantum cases (i.e., the cases that $\rho \in \mathfrak{S}^{p}(\operatorname{Tr}(H)) \subseteq \operatorname{Tr}(H)$, $F(\Xi) \in B(H))$, the probability $\rho(F(\Xi))\left(==_{T r(H)}(\rho, T)_{B(H)}=T_{H}(\rho T)\right)$ is al- 
ways defined $\left(c f .\left(\mathrm{E}_{1}\right)\right)$. That is, the $F(\Xi)$ is always essentially continuous at any $\rho \in \mathfrak{S}^{p}(\operatorname{Tr}(H))$. On the other hand, in the classical cases (i.e., the cases that $\left.\omega \in \Omega, F(\Xi) \in L^{\infty}(\Omega, v)\right)$, it is not guaranteed that $F(\Xi)$ is essentially continuous at $\omega(\in \Omega))$. Thus, put $\mathcal{F}_{\omega_{0}}=\left\{\Xi \in \mathcal{F}: F(\Xi)\right.$ is essentially continuous at $\left.\omega_{0}\right\}$. If $\mathcal{F}_{\omega_{0}}=\mathcal{F}$, the measurement $\mathrm{M}_{L^{\infty}(\Omega, v)}\left(\mathrm{O}:=(X, \mathcal{F}, F), S_{\left[\omega_{0}\right]}\right)$ makes the sample probability space $\left(X, \mathcal{F},[F(\cdot)]\left(\omega_{0}\right)\right)$, which is usual in statistics. Thus, roughly speaking, statistics starts from "sample probability space", on the other hand, quantum language starts from "measurement".

2): Axiom 1 is the quantitative realization of the spirit: "there is no science without measurements". And, we think that Axiom 1 means the probabilistic interpretation of science since it is a kind of mathematical generalization of Born's probability interpretation of quantum mechanics.

Example 5. [Exact measurement] Consider a basic structure $\left[C(\Omega) \subseteq L^{\infty}(\Omega, v) \subseteq B\left(L^{2}(\Omega, v)\right)\right]$, where $\Omega=[0,2]$ (i.e., the closed interval in the real line $\mathbb{R}$ ) is the state space, and the $v$ is the Lebesgue measure on the Borel $\sigma$-field $\mathcal{B}_{\Omega}$, that is, the smallest $\sigma$-field that contains all open sets in $\Omega$. Define the exact observable $\mathrm{O}_{e}=\left(X(=\Omega), \mathcal{F}\left(=\mathcal{B}_{\Omega}\right), F_{e}\right)$ in $L^{\infty}(\Omega, v)$ such that

$$
\left[F_{e}(\Xi)\right](\omega)=1(\omega \in \Xi \in \mathcal{F}), \quad=0(\omega \notin \Xi \in \mathcal{F}) .
$$

Let $\omega^{0} \in \Omega$. Thus, we have the exact measurement $\mathrm{M}_{L^{\infty}(\Omega, v)}\left(\mathrm{O}_{e}=\left(X, \mathcal{F}, F_{e}\right), S_{\left[\omega^{0}\right]}\right)$. Then we have the following statement

- Let $D(\subseteq X=\Omega)$ be any open set such that $\omega^{0} \in D$. Then Axiom 1 says that the probability that the measured value $x(\in X)$ obtained by the measurement $\mathrm{M}_{L^{\infty}(\Omega, v)}\left(\mathrm{O}_{e}=\left(X, \mathcal{F}, F_{e}\right), S_{\left[\omega^{0}\right]}\right)$ belongs to $D$ is given by 1 .

This implies that $x=\omega^{0}$, since $D$ is arbitrary open set such that $\omega^{0} \in D$. Also, it should be noted that $F_{e}(\Xi)$ is not essentially continuous at $\omega^{0}$ if $\omega^{0} \in \partial(\Xi)$, i.e., the boundary of $\Xi$. Thus, $\left(X, \mathcal{F},\left[F_{e}(\cdot)\right]\left(\omega^{0}\right)\right)$ is not always a probability space. However, note that there exists a probability space $(X, \mathcal{F}, \mu)$ such that $\left[F_{e}(\Xi)\right]\left(\omega^{0}\right)=\mu(\Xi) \quad\left(\forall \Xi(\in \mathcal{F})\right.$ such that $\left.\omega^{0} \notin \partial(\Xi)\right)$, though the uniqueness is not guaranteed.

Next, we explain Axiom 2. Let $\left[\mathcal{A}_{1}, \mathcal{N}_{1}\right]_{B\left(H_{1}\right)}$ and $\left[\mathcal{A}_{2}, \mathcal{N}_{2}\right]_{B\left(H_{2}\right)}$ be basic structures. A continuous linear operator $\Phi_{1,2}: \mathcal{N}_{2}$ (with weak ${ }^{*}$ topology) $\rightarrow \mathcal{N}_{1}$ (with weak ${ }^{*}$ topology) is called a Markov operator, if it satisfies that 1): $\Phi_{1,2}\left(F_{2}\right) \geq 0$ for any non-negative element $F_{2}$ in $\left.\mathcal{N}_{2}, 2\right)$ : $\Phi_{1,2}\left(I_{2}\right)=I_{1}$, where $I_{k}$ is the identity in $\mathcal{N}_{k},(k=1,2)$. In addition to the above 1) and 2), we assume that $\Phi_{1,2}\left(\mathcal{A}_{2}\right) \subseteq \mathcal{A}_{1}$ and $\sup \left\{\left\|\Phi_{1,2}\left(F_{2}\right)\right\|_{\mathcal{A}_{1}} \mid F_{2} \in \mathcal{A}_{2}\right.$ such that $\left.\left\|F_{2}\right\|_{\mathcal{A}_{2}} \leq 1\right\}=1$. 
It is clear that the dual operator $\Phi_{1,2}^{*}: \mathcal{A}_{1}^{*} \rightarrow \mathcal{A}_{2}^{*}$ satisfies that $\Phi_{1,2}^{*}\left(\mathfrak{S}^{m}\left(\mathcal{A}_{1}^{*}\right)\right) \subseteq \mathfrak{S}^{m}\left(\mathcal{A}_{2}^{*}\right)$. If it holds that $\Phi_{1,2}^{*}\left(\mathfrak{S}^{p}\left(\mathcal{A}_{1}^{*}\right)\right) \subseteq \mathfrak{S}^{p}\left(\mathcal{A}_{2}^{*}\right)$, the $\Phi_{1,2}$ is said to be deterministic. If it is not deterministic, it is said to be non-deterministic. Also note that, for any observable $\mathrm{O}_{2}:=\left(X, \mathcal{F}, F_{2}\right)$ in $\mathcal{N}_{2}$, the $\left(X, \mathcal{F}, \Phi_{1,2} F_{2}\right)$ is an observable in $\mathcal{N}_{1}$.

Definition 6. [Sequential causal operator; Heisenberg picture of causality] Let $(T, \leq)$ be a tree like semi-ordered set such that $t_{1} \leq t_{3}$ and $t_{2} \leq t_{3}$ implies $t_{1} \leq t_{2}$ or $t_{2} \leq t_{1}$. The family $\left\{\Phi_{t_{1}, t_{2}}: \mathcal{N}_{t_{2}} \rightarrow \mathcal{N}_{t_{1}}\right\}_{\left(t_{1}, t_{2}\right) \in T_{\leqq}^{2}}$ is called a sequential causal operator, if it satisfies that

1) For each $t(\in T)$, a basic structure $\left[\mathcal{A}_{t} \subseteq \mathcal{N}_{t} \subseteq B\left(H_{t}\right)\right]$ is determined.

2) For each $\left(t_{1}, t_{2}\right) \in T_{\leqq}^{2}$, a causal operator $\Phi_{t_{1}, t_{2}}: \mathcal{N}_{t_{2}} \rightarrow \mathcal{N}_{t_{1}}$ is defined such as $\Phi_{t_{1}, t_{2}} \Phi_{t_{2}, t_{3}}=\Phi_{t_{1}, t_{3}} \quad\left(\forall\left(t_{1}, t_{2}\right), \forall\left(t_{2}, t_{3}\right) \in T_{\leqq}^{2}\right)$. Here, $\Phi_{t, t}: \mathcal{N}_{t} \rightarrow \mathcal{N}_{t}$ is the identity operator.

Now we can propose Axiom 2 (i.e., causality). (For details, see ref. [18].)

Axiom 2 [Causality]; For each $t$ ( $\in T=$ "tree like semi-ordered set"), consider the basic structure:

$$
\left[\mathcal{A}_{t} \subseteq \mathcal{N}_{t} \subseteq B\left(H_{t}\right)\right]
$$

Then, the chain of causalities is represented by a sequential causal operator $\left\{\Phi_{t_{1}, t_{2}}: \mathcal{N}_{t_{2}} \rightarrow \mathcal{N}_{t_{1}}\right\}_{\left(t_{1}, t_{2}\right) \in T_{\leqq}^{2}}$.

When parameters $t_{1}, t_{2}\left(t_{1}<t_{2}\right)$ are regarded as time, we usually consider that a causal operator $\Phi_{t_{1}, t_{2}}: \mathcal{N}_{t_{2}} \rightarrow \mathcal{N}_{t_{1}}$ represents "causality". Thus, this axiom gives an answer to "What is causality?". That is, we consider that, if Axiom 2 is used in the quantum linguistic representation of a phenomenon, causality exists in the phenomenon.

\subsection{The Linguistic Copenhagen Interpretation (=The Manual to Use Axioms 1 and 2)}

It is well known (cf. ref. [25]) that the Copenhagen interpretation of quantum mechanics has not been established yet. For example, about the right or wrong of the wave function collapse, opinions are divided in the Copenhagen interpretation. Thus, the Copenhagen interpretation is often called "so-call Copenhagen interpretation". However, we believe that the linguistic Copenhagen interpretation of quantum language (B) (i.e., both quantum $\left(\mathrm{B}_{1}^{\prime}\right)$ and classical $\left(\mathrm{B}_{2}^{\prime}\right)$ ) is uniquely determined. For example, for the quantum linguistic opinion about the wave function collapse, see ref. [13] or $\$ 11.2$ in ref. [18]. As mentioned in $\left(B_{1}\right)$, we believe that the linguistic Copenhagen interpretation is the true figure of so called Copenhagen interpretation.

Now we explain the linguistic Copenhagen interpretation in what follows. In the above, Axioms 1 and 2 are kinds of spells, (i.e., incantation, magic words, metaphysical statements), and thus, it is nonsense to verify them experimentally. Therefore, what we should do is not "to understand" but "to use". After learning 
Axioms 1 and 2 by rote, we have to improve how to use them through trial and error. We may do well even if we do not know the linguistic Copenhagen interpretation (=the manual to use Axioms 1 and 2). However, it is better to know the linguistic Copenhagen interpretation, if we would like to make progress quantum language early. We believe that the linguistic Copenhagen interpretation is the true Copenhagen interpretation, which does not belong to physics.

The essence of the manual is as follows: In Figure 2, we remark:

$\left(\mathrm{F}_{1}\right) \otimes$ : it suffices to understand that interfere "is, for example, apply light".

(D: perceive the reaction.

That is, "measurement" is characterized as the interaction between "observer" and "measuring object (= matter)". However,

$\left(\mathrm{F}_{2}\right)$ in measurement theory (=quantum language), "interaction" must not be emphasized.

Therefore, in order to avoid confusion, it might better to omit the interaction “® and $($ )" in Figure 2.

After all, we think that:

$\left(\mathrm{F}_{3}\right)$ it is clear that there is no measured value without observer (i.e., "I", "mind"). Thus, we consider that measurement theory is composed of three key-words: "measured value”, “observable”, “state” (cf. \$3.1(p.63) in [18]).

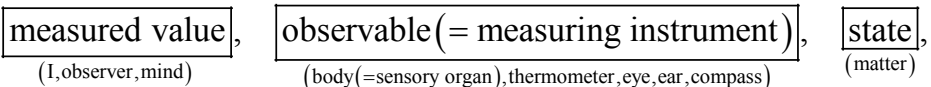

Hence, quantum language is based on dualism, i.e., a kind of mind-matter dualism.

The linguistic Copenhagen interpretation says that

$\left(G_{1}\right)$ Only one measurement is permitted. And therefore, the state after a measurement is meaningless since it cannot be measured any longer. Thus, the collapse of the wavefunction is prohibited (cf. ref. [13]; projection postulate). We are not concerned with anything after measurement. Strictly speaking, the phrase after the measurement should not be used. Also, the causality should be assumed only in the side of system, however, a state never moves. Thus, the Heisenberg picture should be adopted, and thus, the Schrödinger picture should be prohibited.

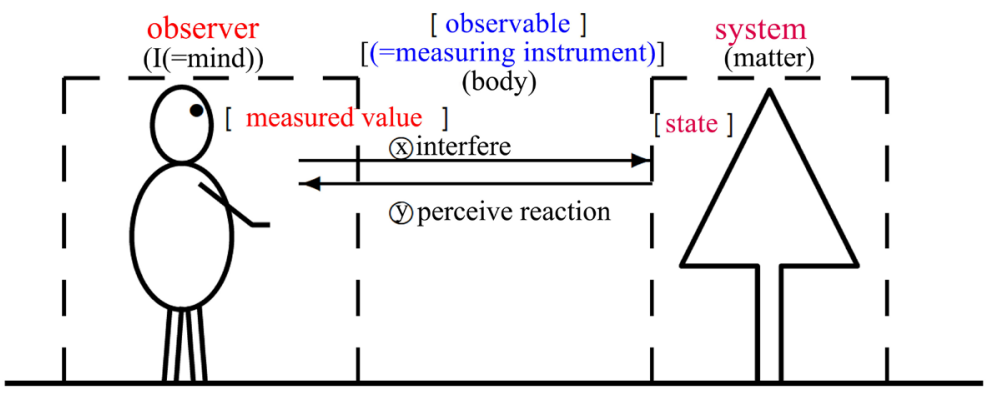

Figure 2. [Descartes Figure]: Image of measurement $(=x+y)$ in mind-matter dualism. 
$\left(G_{2}\right)$ "Observer" (="I") and "system" are completely separated in order not to make self-reference propositions appear. Hence, the measurement $\mathrm{M}_{\mathcal{N}}\left(\mathrm{O}=(X, \mathcal{F}, F), S_{[\rho]}\right)$ does not depend on the choice of observers. That is, any proposition (except Axiom 1) in quantum language is not related to "observer"(="I"), therefore, there is no "observer's space and time" in quantum language. And thus, it does not have tense (i.e., past, present, future).

$\left(\mathrm{G}_{3}\right)$ there is no probability without measurements (cf. Bertrand's paradox (in $\$ 9.12$ of ref. [18])).

$\left(\mathrm{G}_{4}\right)$ Leibniz's relationalism concerning space-time (e.g., time should be regarded as a parameter), (cf. ref. [17]).

$\left(\mathrm{G}_{5}\right)$ A family of measurements $\left\{\mathrm{M}_{\mathcal{N}_{i}}\left(\mathrm{O}_{i}:=\left(X_{1}, \mathcal{F}_{i}, F_{i}\right), S_{\left[\rho_{i}\right]}\right): i=1,2,3, \cdots\right\}$ is realized as the paralell measurement

$\mathrm{M}_{\bigotimes_{i=1}^{\infty} \mathcal{N}_{i}}\left(\otimes_{i=1}^{\infty} \mathrm{O}_{i}:=\left(\times_{i=1}^{\infty} X_{1}, \bigotimes_{i=1}^{\infty} F_{i}, \bigotimes_{i=1}^{\infty} F_{i}\right), S_{\left[\otimes_{i=1}^{\infty} \rho_{i}\right]}\right)$ (cf. Definition 8 later). For details about the tensor product “ $\otimes_{i=1}^{\infty}$ ”, see ref. [18].

and so on.

Remark 7. 1): In ref. [3] (1991), we proposed the mathematical formulation of Heisenberg's uncertainty relation. However, so-called Copenhagen interpretation is not firm (cf. ref. [25]). Thus, in order to understand our work (i.e., Heisenberg's uncertainty relation) deeply, we proposed the linguistic Copenhagen interpretation (=quantum language) as the true Copenhagen interpretation. For details of Heisenberg’s uncertain relation, see $\$ 4.3$ in ref. [18].

2 ): We consider that the above $\left(G_{1}\right)$ is closely related to Kolmogorov's extension theorem (cf. ref. [26]), which says that only one probability space is permitted. For details, see $\$ 4.1$ in ref. [18].

3): The formula (1) says that scientific explanation is to explain phenomena in terms of "measurement" (Axiom 1) and "causality" (Axiom 2). If we are allowed to use the famous metaphor of Kant's Copernican revolution, to do familiar sciences is to see this world through colored glasses of measurement and causality (cf. [17]), or to use the metaphor of Wittgenstein's saying, the limits of quantum language are the limits of familiar science. Therefore, the explanation problem of scientific philosophy is automatically clarified in quantum language.

4): Violating the linguistic Copenhagen interpretation $\left(G_{2}\right)$, we have many paradoxes of self-reference type such as "brain in a vat", "five-minute hypothesis", "I think, therefore I am”, “McTaggart’s paradox”. Cf. ref. [17] or $\$ 10.8$ in ref. [18].

5): We want to understand that Zeno's paradox is not a problem concerning geometric series or spatial division, but the problem concerning the worldview. That is, "Propose a certain scientific worldview, in which Zeno's paradox should be studied!" That is because we think that there is no scientific argument without scientific language ( $\approx$ scientific worldview). And our answer ( $c f . \$ 14.4$ in ref. [18]) is "If Zeno's paradox is a problem in science, it should be studied in quantum language". That is because our assertion is "Quantum language is the language of science". Also, Monty Hall problem, two envelope problem, three pris- 
oners problem etc. are not only mathematical puzzles but also profound problems in quantum language (cf. refs. [11] [18]).

As the further explanation of parallel measurement in the linguistic Copenhagen interpretation $\left(G_{5}\right)$, we have to add the following definition.

Definition 8. [Parallel measurement (cf. [18])] Though the parallel measurement can be defined in both classical and quantum systems, we, for simplicity, devote ourselves to classical systems as follows. Let $\left[C(\Omega) \subseteq L^{\infty}(\Omega, v) \subseteq B\left(L^{2}(\Omega, v)\right)\right]$ be a classical basic structure, where we assume, for simplicity, that $\Omega$ is compact space and $v$ is a measure such that $v(\Omega)=1$ and $v(D)>0 \quad(\forall$ open set $D \subseteq \Omega)$. Consider a family of measurements $\left\{\mathrm{M}_{L^{\infty}(\Omega, v)}\left(\mathrm{O}_{i}:=\left(X_{i}, \mathcal{F}_{i}, F_{i}\right), S_{\left[\omega_{i}\right]}\right) \mid i=1,2, \cdots, N\right\}$. However, the linguistic Copenhagen interpretation $\left(G_{1}\right)$ says "Only one measurement is permitted". Therefore, instead of the family of measurements, we consider the parallel measurement $\otimes_{i=1}^{N} \mathrm{M}_{L^{\infty}(\Omega, v)}\left(\mathrm{O}_{i}:=\left(X_{i}, \mathcal{F}_{i}, F_{i}\right), S_{\left[\omega_{i}\right]}\right)$, which is defined by

$$
\begin{aligned}
& \bigotimes_{i=1}^{N} \mathrm{M}_{L^{\infty}(\Omega, v)}\left(\mathrm{O}_{i}:=\left(X_{i}, \mathcal{F}_{i}, F_{i}\right), S_{\left[\omega_{i}\right]}\right) \\
& =\mathrm{M}_{L^{\infty}\left(x_{i=1}^{N} \Omega, \otimes_{i=1}^{N} \nu\right)}\left(\otimes_{i=1}^{N} \mathrm{O}_{i}:=\left(\underset{i=1}{\times} X_{i}, \bigotimes_{i=1}^{N} \mathcal{F}_{i}, \otimes_{i=1}^{N} F_{i}\right), S_{\left[\left(\omega_{1}, \omega_{2}, \cdots, \omega_{N}\right)\right]}\right)
\end{aligned}
$$

where $\times_{i=1}^{N} \Omega$ is the finite product compact space of $\Omega s, \otimes_{i=1}^{N} v$ is the infinite product probability of $v$ s. Also, $\bigotimes_{i=1}^{N} \mathcal{F}_{i}\left(\subseteq \mathcal{P}\left(x_{i=1}^{N} X_{i}\right)\right)$ is the finite product $\sigma$-field, i.e., the smallest $\sigma$-field that includes

$$
\left\{\underset{i=1}{N} \Xi_{i} \mid \Xi_{i} \in \mathcal{F}_{i}\right\}
$$

And further, define the observable $\otimes_{i=1}^{N} F_{i}$ in $L^{\infty}\left(\times_{i=1}^{N} \Omega, \otimes_{i=1}^{N} v\right)$ which satisfies that

$$
\left[\left(\otimes_{i=1}^{N} F_{i}\right)\left(\underset{i=1}{\times} \Xi_{i}\right)\right]\left(\omega_{1}, \omega_{2}, \cdots, \omega_{N}\right)=\underset{i=1}{\times}\left[F_{i}\left(\Xi_{i}\right)\right]\left(\omega_{i}\right) \quad \forall \Xi_{i} \in \mathcal{F}_{i}, \omega_{i} \in \Omega
$$

Then, Axiom 1 [measurement] says that

$(\mathrm{H})$ the probability that a measured value obtained by the parallel measurement $\otimes_{i=1}^{N} \mathrm{M}_{L^{\infty}(\Omega, v)}\left(\mathrm{O}_{i}:=\left(X_{i}, \mathcal{F}_{i}, F_{i}\right), S_{\left[\omega_{i}\right]}\right)$ belongs to $\times_{i=1}^{N} \Xi_{i}$ is given by $\times_{i=1}^{N}\left[F_{i}\left(\Xi_{i}\right)\right]\left(\omega_{i}\right)$, if $F_{i}\left(\Xi_{i}\right)$ is essentially continuous at $\omega_{i} \quad(\forall i=1,2, \cdots, N)$.

Remark 9. The above finite parallel measurement can be generalized to the case that the index set $\Lambda$ is infinite. That is,

$$
\begin{aligned}
& \underset{\lambda \in \Lambda}{\otimes} \mathrm{M}_{L^{\infty}(\Omega, v)}\left(\mathrm{O}_{\lambda}:=\left(X_{\lambda}, \mathcal{F}_{\lambda}, F_{\lambda}\right), S_{\left[\omega_{\lambda}\right]}\right) \\
& =\mathrm{M}_{L^{\infty}\left(x_{\lambda \in \Lambda} \Omega, \otimes_{\lambda \in \Lambda} v\right)}\left(\otimes_{\lambda \in \Lambda} \mathrm{O}_{\lambda}:=\left(\underset{\lambda \in \Lambda}{\times} X_{\lambda}, \bigotimes_{\lambda \in \Lambda} \mathcal{F}_{\lambda}, \otimes_{\lambda \in \Lambda} F_{\lambda}\right), S_{\left[\left(\omega_{\lambda}\right)_{\lambda \in \Lambda}\right]}\right)
\end{aligned}
$$

The existence of the parallel measurement is guaranteed in both classical and quantum systems. Cf. $\$ 4.2$ in ref. [18]. It is not so difficult to extend the above finite parallel measurements to infinite parallel measurements for mathemati- 
cians. However, in this paper, we are not concerned with the infinite parallel measurement. That is because our concern is not mathematics but foundations of philosophy of science.

Here we add the following definition, which will be used in [Step (III)] in Section 3.2.

Definition 10. [Implication: $c f$. refs. [4] [18]] Consider a basic structure:

$$
[\mathcal{A} \subseteq \mathcal{N} \subseteq B(H)]
$$

Let $\mathrm{O}_{1}=\left(X_{1}, \mathcal{F}_{1}, F_{1}\right)$ and $\mathrm{O}_{2}=\left(X_{2}, \mathcal{F}_{2}, F_{2}\right)$ be observables in $\mathcal{N}$. Let $\mathrm{O}_{12}=\left(X_{1} \times X_{2}, \mathcal{F}_{1} \otimes \mathcal{F}_{2}, F_{12}\right)$ be an observable such that $F_{1}\left(\Xi_{1}\right)=F_{12}\left(\Xi_{1} \times X_{2}\right)$ and $F_{2}\left(\Xi_{2}\right)=F_{12}\left(X_{1} \times \Xi_{2}\right) \quad\left(\forall \Xi_{1} \in \mathcal{F}_{1}, \Xi_{2} \in \mathcal{F}_{2}\right)$. Let $\rho \in \mathfrak{S}^{p}\left(\mathcal{A}^{*}\right), \quad \Gamma_{1} \in \mathcal{F}_{1}$, $\Gamma_{2} \in \mathcal{F}_{2}$. Then, if it holds that

$$
\rho\left(F_{12}\left(\Gamma_{1} \times\left(X_{2} \backslash \Gamma_{2}\right)\right)\right)=0
$$

this is denoted by

$$
\left[\mathrm{O}_{1} ; \Gamma_{1}\right]_{\mathrm{M}_{\mathcal{N}}\left(\mathrm{O}_{12}, \mathrm{~S}_{[\rho]]}\right)}^{\Rightarrow}\left[\mathrm{O}_{2} ; \Gamma_{2}\right] \text { or, equivalently }\left[\mathrm{O}_{1} ; X_{1} \backslash \Gamma_{1}\right]_{\mathrm{M}_{\mathcal{N}}\left(\mathrm{O}_{12}, \mathrm{~S}_{[\rho]}\right]}\left[\mathrm{O}_{2} ; X_{2} \backslash \Gamma_{2}\right]
$$

That is because the probability that a measured value $\left(x_{1} \cdot x_{2}\right)$ obtained by the measurement $\mathrm{M}_{\mathcal{N}}\left(\mathrm{O}_{12}:=\left(X_{1} \times X_{2}, \mathcal{F}_{1} \otimes \mathcal{F}_{2}, F_{12}\right), S_{[\rho]}\right)$ belongs to $\Gamma_{1} \times\left(X_{2} \backslash \Gamma_{2}\right)$ is equal to 0 .

Remark 11. [Syllogism] Using Definition 10, we showed that

- Syllogism always holds in classical systems (cf. ref. [4])

- Syllogism does not always hold in quantum systems (cf. ref. [9], \$8.7 in ref. [18])

\subsection{Inference; Fisher's Maximum Likelihood Method}

We begin with the following notation:

Notation 12. $\left[\mathrm{M}_{L^{\infty}(\Omega, v)}\left(\mathrm{O}, S_{[*]}\right)\right]$ : Consider a measurement

$\mathrm{M}_{L^{\infty}(\Omega, v)}\left(\mathrm{O}:=(X, \mathcal{F}, F), S_{\left[\omega_{0}\right]}\right)$ formulated in the basic structure

$\left[C(\Omega) \subseteq L^{\infty}(\Omega, v) \subseteq B\left(L^{2}(\Omega, v)\right)\right]$. Here, note that

$\left(\mathrm{I}_{1}\right)$ in most cases that the measurement $\mathrm{M}_{L^{\infty}(\Omega, v)}\left(\mathrm{O}:=(X, \mathcal{F}, F), S_{\left[\omega_{0}\right]}\right)$ is taken, it is usual to think that the state $\omega_{0}(\in \Omega)$ is unknown.

That is because

$\left(\mathrm{I}_{2}\right)$ the measurement $\mathrm{M}_{L^{\infty}(\Omega, v)}\left(\mathrm{O}, S_{\left[\omega_{0}\right]}\right)$ may be taken in order to know the state $\omega_{0}$.

Therefore, when we want to stress that we do not know the state $\omega_{0}$, the measurement $\quad \mathrm{M}_{L^{\infty}(\Omega, v)}\left(\mathrm{O}:=(X, \mathcal{F}, F), S_{\left[\omega_{0}\right]}\right) \quad$ is often denoted by $\mathrm{M}_{L^{\infty}(\Omega, v)}\left(\mathrm{O}:=(X, \mathcal{F}, F), S_{\left[*^{*}\right]}\right)$.

Theorem 13. [Inference; Fisher's maximum likelihood method (cf. ref. [5] or $\$ 5.2$ in ref. [18]] For simplicity, assume that $X$ is finite set. Assume that the 
measured value $x(\in X)$ is obtained by the measurement

$\mathrm{M}_{L^{\infty}(\Omega, v)}\left(\mathrm{O}:=\left(X, 2^{X}, F\right), S_{[*]}\right)$. Then, the unknown state $[*]$ can be inferred to be $\omega_{0}(\in \Omega)$ such that

$$
[F(\{x\})]\left(\omega_{0}\right)=\max _{\omega \in \Omega}[F(\{x\})](\omega)
$$

Proof. It is an easy consequence of Axiom 1 (cf. $\$ 5.2$ in ref. [18]).

Remark 14. [Inference and Control $c f$. $\$ 5.2$ in ref. [18]] The inference problem is characterized as the reverse problem of measurements. That is, we consider that

$\left(\mathrm{J}_{1}\right)\left(\right.$ state $\omega_{0}$, observable $\left.\mathrm{O}\right) \frac{\mathrm{M}_{L^{\infty}(\Omega, v)}\left(\mathrm{O}:=\left(X, 2^{X}, F\right), S_{\left[\omega_{0}\right]}\right)}{\text { measurement }(\mathrm{Axiom} 1)}$ measured value $x_{0}$

On the other hand

$\left(\mathrm{J}_{2}\right)$ (measured value $x_{0}$, observable $\left.\mathrm{O}\right) \frac{\mathrm{M}_{L^{\infty}(\Omega, v)}\left(\mathrm{O}:=\left(X, 2^{X}, F\right), S_{[?]}\right)}{\text { inference( (reverse Axiom 1) }}$ state $\omega_{0}$

Thus, $\left(\mathrm{J}_{1}\right)$ and $\left(\mathrm{J}_{2}\right)$ are in reverse problem.

Also, we note, from the mathematical point of view, that inference problem $\left(J_{3}\right)$ and control problem $\left(\mathrm{J}_{4}\right)$ are essentially the same as follows.

$\left(\mathrm{J}_{3}\right)$ [Inference problem; statistics]: when measured value $x_{0}$ is obtained, infer the unknown state $\omega_{0}$ !

and

$\left(\mathrm{J}_{4}\right)$ [Control problem; dynamical system theory]: Settle the state $\omega_{0}$ such that measured value $x_{0}$ will be obtained!

Thus, we think, from the theoretical point of view, that statistics and dynamical system theory are essentially the same. Thus, we consider that statistics (=dynamical system theory) is the mathematical representation of classical mechanical worldview. On the other hand, quantum language is regarded as the mathematical representation of quantum mechanical worldview.

\section{Hempel's Raven Paradox in the Linguistic Quantum Mechanical Worldview}

\subsection{What Is Hempel's Raven Paradox?}

Although all results mentioned in this paper hold in both classical and quantum systems, we, for simplicity, devote ourselves to classical systems.

In this section we discuss Hempel's raven paradox (cf. ref. [1]) in the linguistic quantum mechanical worldview. There may no consensus among philosophers on the problem "What is Hempel's raven paradox?". Some people may even think there is no paradox in Hempel's raven problem. Thus, we mention our opinion about "What is Hempel's raven paradox?" in what follows. Let $U$ be the universal set of all birds. Let $B(\subseteq U)$ be a set of all black birds. Let $R(\subseteq U)$ be a set of all ravens. Thus, the statement: "any raven is black" is logically denoted by

$\left(\mathrm{K}_{1}\right)$ "Any raven is black": $(\forall x)[x \in R \rightarrow x \in B]$ i.e., $R \subseteq B \subseteq U$,

Its contraposition is denoted by

$\left(\mathrm{K}_{2}\right)$ "Every non-black bird is a nonraven": $(\forall x)[x \in U \backslash B \rightarrow x \in U \backslash R]$ i.e., 
$U \backslash B \subseteq U \backslash R$

Of course, the two $\left(\mathrm{K}_{1}\right)$ and $\left(\mathrm{K}_{2}\right)$ are equivalent. However, If $\left(\mathrm{K}_{1}\right)$ and $\left(\mathrm{K}_{2}\right)$ are equivalent, then we have the following questions (i.e., raven problem):

$\left(\mathrm{K}_{3}\right)$ Why is the actual verification of $\left(\mathrm{K}_{2}\right)$ much more difficult than the actual verification of $\left(\mathrm{K}_{1}\right)$ ?

$\left(\mathrm{K}_{4}\right)$ Why can the truth of $\left(\mathrm{K}_{1}\right)$ : "any raven is black" be known by $\left(\mathrm{K}_{2}\right)$, i.e., without seeing a raven also at once?

Some may think that these are nonsense questions. However, in this section, we clarify the true meaning of $\left(\mathrm{K}_{3}\right)$ and $\left(\mathrm{K}_{4}\right)$ in the linguistic quantum mechanical worldview. And further, we conclude that Hempel's raven paradox may suggest the importance of measurement in science, that is, the language of science is not logic but quantum language.

Remark 15. Throughout this section, we assume that $U$ (the universal set of all birds) is finite, i.e., $\#[U]<\infty$ (where $\#[S]$ is denoted by the number of elements of a set $S)$. Some may misunderstand that the questions $\left(\mathrm{K}_{3}\right)$ and $\left(\mathrm{K}_{4}\right)$ are due to the fact such that $\#[R] \ll \#[U \backslash B] \approx \infty$. This is wrong. That is, as shown in the next section, the questions arise even in the case that $\#[U \backslash B] \ll \#[R]$. In order to avoid misunderstanding, we assume that $U$ is a finite set.

\subsection{The Measurement Theoretical Answer to the Raven Paradox}

Although most results mentioned in Sections 3 6 hold in both classical and quantum systems, we, for simplicity, devote ourselves to classical systems:

Let $U$ be the universal set of all birds. Let $B(\subseteq U)$ be a set of all black birds. Let $R$ be the set of all ravens. Assume that $U$ is finite. Thus, "Any raven is black" is logically denoted by

$\left(\mathrm{L}_{1}\right) \quad R \subseteq B \subseteq U$, i.e., $(\forall x)[x \in R \rightarrow x \in B]$; "any raven is black"

This is logically equivalent to the following $\left(\mathrm{L}_{2}\right)$ and $\left(\mathrm{L}_{3}\right)$ : (i.e., $\left(\mathrm{L}_{1}\right) \Leftrightarrow\left(\mathrm{L}_{2}\right)$ $\left.\Leftrightarrow\left(\mathrm{L}_{3}\right)\right)$

$\left(\mathrm{L}_{2}\right) \quad[U \backslash B] \subseteq[U \backslash R] \subseteq U$, i.e., $\quad(\forall x)[x \in U \backslash B \rightarrow x \in U \backslash R] \quad ; \quad$ "every non-black bird is a nonraven"

$\left(\mathrm{L}_{3}\right) \quad R \cap[U \backslash B]=\varnothing$, i.e., $(\neg \exists x)[[x \in R] \wedge[x \in U \backslash B]]$; “a non-black raven does not exist"

In what follows we try to explain the measurement theoretical (i.e., dualistic) representations of the logical (or set theoretical) statements $\left(\mathrm{L}_{1}\right),\left(\mathrm{L}_{2}\right)$ and $\left(\mathrm{L}_{3}\right)$ :

Let $\Omega$ be the state space. Without loss of generality (and, for simplicity), the state space $\Omega$ is assumed to be a compact space. Let $v$ be a measure on $\Omega$ such that $v(\Omega)=1$ and $v(V)>0 \quad(\forall$ open set $V(\subseteq \Omega))$. Thus we have the following classical basic structure:

$$
\left[C(\Omega) \subseteq L^{\infty}(\Omega, v) \subseteq B\left(L^{2}(\Omega, v)\right)\right]
$$

Consider the state subspaces $\Omega_{R}(\subseteq \Omega)$ and $\Omega_{B}(\subseteq \Omega)$ such that $\Omega_{R} \subseteq \Omega_{B}$. And define the state map $\tilde{\omega}: U \rightarrow \Omega$, that is, the state of a bird 
$t(\in U)$ is denoted by $\tilde{\omega}(t)(\in \Omega)$. And further assume that

$$
\tilde{\omega}(R) \subseteq\left(\Omega_{R}\right)^{\circ}, \quad \tilde{\omega}(B \backslash R) \subseteq\left(\Omega_{B} \backslash \Omega_{R}\right)^{\circ}, \quad \tilde{\omega}(U \backslash B) \subseteq\left(\Omega \backslash \Omega_{B}\right)^{\circ},
$$

where $D^{\circ}$ is the interior of $D(\subseteq \Omega)$, i.e.,

$D^{\circ} \equiv \bigcup\{O \mid O \subseteq D(\subseteq \Omega), O$ is open $\}$. This condition is necessary for essentially continuity" in Axiom 1 (also, see Remark 4).

Remark 16. Without loss of generality, we can assume that $\Omega=\left\{\omega_{1}, \omega_{2}, \omega_{3}\right\}$ (with discrete topology), $\Omega_{R}=\left\{\omega_{1}\right\}, \Omega_{B}=\left\{\omega_{1}, \omega_{2}\right\}$ and

$$
\tilde{\omega}(t)=\omega_{1}(t \in R), \quad=\omega_{2}(t \in B \backslash R), \quad=\omega_{3}(t \in U \backslash B)
$$

However, we devote ourselves to the above general situation.

[Step (I)]: The measurement theoretical representation of $\left(\mathrm{L}_{1}\right)$; Any raven is black"

Now let us study the measurement theoretical representation of $\left(\mathrm{L}_{1}\right)$ : "Any raven is black". Define the observable $\mathrm{O}_{B}=\left(X, 2^{X}, F\right)$ in $L^{\infty}(\Omega, v)$ such that

$$
\begin{aligned}
& X=\{b, \bar{b}\}, \\
& {[F(\{b\})](\omega)=1 \quad\left(\text { if } \omega \in \Omega_{B}\right), \quad[F(\{b\})](\omega)=0 \quad\left(\text { if } \omega \in \Omega \backslash \Omega_{B}\right),} \\
& {[F(\{\bar{b}\})](\omega)=1-[F(\{b\})](\omega)(\omega \in \Omega)}
\end{aligned}
$$

where " $b$ " and " $\bar{b}$ " means "black" and "non-black" respectively.

Now, for any $t \in R\left(\equiv\left\{r_{1}, r_{2}, \cdots, r_{\#[R]}\right\}\right)$, we have the measurement $\mathrm{M}_{L^{\infty}(\Omega, v)}\left(\mathrm{O}_{B}:=\left(X, 2^{X}, F\right), S_{[\tilde{\omega}(t)]}\right)$, i.e., the measurement of whether the raven $t(\in R)$ is black. Axiom 1 [measurement] says that, for any (=arbitrary) raven $t(\in R)$,

$\left(\mathrm{M}_{1}\right)$ the probability that a measured value obtained by the measurement $\mathrm{M}_{L^{\infty}(\Omega, v)}\left(\mathrm{O}_{B}:=\left(X, 2^{X}, F\right), S_{[\tilde{\omega}(t)]}\right)$ is equal to $b(\in X)$ is given by 1 $(\equiv[F(\{b\})](\tilde{\omega}(t))=1)$.

This is one of the measurement theoretical representations of the statement: "Any raven is black".

However, the above term "for any raven" is too logical and not concrete. Thus, we may, by using the parallel measurement $(c f .(\mathrm{H})$ in Definition 8$)$, rewrite the $\left(\mathrm{M}_{1}\right)$ to the following:

$\left(\mathrm{M}_{2}\right)$ the probability that a measured value $\left(x_{t}\right)_{t \in R}\left(\equiv\left(x_{i}\right)_{i=1}^{\#[R]} \in X^{R}\right)$ obtained by the parallel measurement $\otimes_{t \in R} \mathrm{M}_{L^{\infty}(\Omega, v)}\left(\mathrm{O}_{B}:=\left(X, 2^{X}, F\right), S_{[\tilde{\omega}(t)]}\right)$ is equal to $(b)_{t \in R}\left(\equiv(b)_{i=1}^{\sharp[R]} \in X^{R}\right)$ is given by $1\left(\equiv \times_{t \in R}[F(\{b\})](\tilde{\omega}(t))=1\right)$.

which is a formal (i.e., measurement theoretical) expression of the $\left(\mathrm{L}_{1}\right)$. Also, this may mean "All ravens are black" rather than "Any raven is black". For completeness, note that "any (=arbitrary)" and "all" are distinguished in quantum language.

[Step (II)]: The measurement theoretical representation of $\left(\mathrm{L}_{2}\right)$; "Every 
non-black bird is a nonraven"

Here let us study the measurement theoretical representation of $\left(\mathrm{L}_{2}\right)$ : "Every non-black bird is a nonraven". Define the observable $\mathrm{O}_{R}=\left(Y, 2^{Y}, G\right)$ in $L^{\infty}(\Omega, v)$ such that

$$
\begin{aligned}
& \left.\left.[G(\{r\})](\omega)=1 \quad \text { if } \omega \in \Omega_{R}\right), \quad[G(\{r\})](\omega)=0 \quad \text { if } \omega \in \Omega \backslash \Omega_{R}\right), \\
& {[G(\{\bar{r}\})](\omega)=1-[G(\{r\})](\omega)(\omega \in \Omega)}
\end{aligned}
$$

where " $r$ " and " $\bar{r}$ " means "raven" and "nonraven" respectively. Now, for any $t \in U \backslash B$, we have the measurement $\mathrm{M}_{L^{\infty}(\Omega, v)}\left(\mathrm{O}_{R}:=\left(Y, 2^{Y}, F\right), S_{[\tilde{\omega}(t)]}\right)$, i.e., the measurement of whether the non-black bird $t \in U \backslash B$ is a nonraven. Axiom 1 [measurement] says that, for any non-black bird $t \in U \backslash B$,

$\left(\mathrm{N}_{1}\right)$ the probability that a measured value obtained by the measurement $\mathrm{M}_{L^{\infty}(\Omega, v)}\left(\mathrm{O}_{R}:=\left(Y, 2^{Y}, F\right), S_{[\tilde{\omega}(t)]}\right) \quad$ is equal to $\bar{r}(\in Y)$ is given by 1 $(\equiv[G(\{\bar{r}\})](\tilde{\omega}(t))=1)$.

This is one of the measurement theoretical representations of the statement: "Every non-black bird is a nonraven". However, the above term "for any non-black bird" is too logical and not concrete. Thus, we may, by using the parallel measurement $(c f .(\mathrm{H})$ in Definition 8$)$, rewrite the $\left(\mathrm{N}_{1}\right)$ to the following:

$\left(\mathrm{N}_{2}\right)$ the probability that a measured value $\left(y_{t}\right)_{t \in U \backslash B}\left(\in Y^{U \backslash B}\right)$ obtained by the parallel measurement $\otimes_{t \in U \backslash B} \mathrm{M}_{L^{\infty}(\Omega, v)}\left(\mathrm{O}_{R}:=\left(Y, 2^{Y}, F\right), S_{[\tilde{\omega}(t)]}\right)$ is equal to $(\bar{r})_{t \in U \backslash B}$ is given by 1 ,

which is a formal (i.e., measurement theoretical) expression of the $\left(\mathrm{L}_{2}\right)$. Also, this may mean "All the birds non-black are nonravens" rather than "Any non-black bird is a nonraven".

Note that the argument of [Step(I)] and that of [Step(II)] are essentially the same (i.e., the role of $R$ and the role of $U \backslash B$ are symmetrical). However, the following [Step(III)] is different from them.

[Step (III)]: The measurement theoretical representation of $\left(\mathrm{L}_{3}\right)$; “A non-black raven does not exist"

Let $\mathrm{O}_{B}:=\left(X, 2^{X}, F\right)$ and $\mathrm{O}_{R}:=\left(Y, 2^{Y}, G\right)$ be as in [Step (I)] and [Step (II)] respectively.

Define the observable $\mathrm{O}_{B R}:=\left(X \times Y=\{(b, r),(b, \bar{r}),(\bar{b}, r),(\bar{b}, \bar{r})\}, 2^{X \times Y}, H\right)$ in $L^{\infty}(\Omega, v)$ such that, for any $\omega \in \Omega$,

$$
\left\{\begin{array}{l}
{[H(\{(b, r)\})](\omega)=[F(\{b\})](\omega) \cdot[G(\{r\})](\omega)=[G(\{r\})](\omega)} \\
{[H(\{(b, \bar{r})\})](\omega)=[F(\{b\})](\omega) \cdot[G(\{\bar{r}\})](\omega)} \\
{[H(\{(\bar{b}, r)\})](\omega)=[F(\{\bar{b}\})](\omega) \cdot[G(\{r\})](\omega)=0} \\
{[H(\{(\bar{b}, \bar{r})\})](\omega)=[F(\{\bar{b}\})](\omega) \cdot[G(\{\bar{r}\})](\omega)=[F(\{\bar{b}\})](\omega)}
\end{array}\right.
$$

Then we see:

$\left(\mathrm{O}_{1}\right)$ Let $t$ be any bird in $U$. Assume that a measured value $(x, y) \in X \times Y$ is 
obtained by the measurement $\mathrm{M}_{L^{\infty}(\Omega, v)}\left(\mathrm{O}_{B R}:=\left(X \times Y, 2^{X \times Y}, H\right), S_{[\tilde{\omega}(t)]}\right)$. Axiom 1 [measurement] says that the probability that $(x, y)=(\bar{b}, r)$ is equal to 0 $(\equiv[H(\{(\bar{b}, r)\})](\tilde{\omega}(t))=0)$. When we paraphrase, Axiom 1 [measurement] says that the probability that $(x, y) \in\{(b, r),(b, \bar{r}),(\bar{b}, \bar{r})\}$ is equal to 1 .

This, of course, means “ $A$ non-black raven does not exist'. Or, using Definition 10 [implication], we can describe

$$
\begin{aligned}
& {\left[\mathrm{O}_{B} ;\{\bar{b}\}\right]_{\mathrm{M}_{L^{\infty}(\Omega, v)}\left(\mathrm{O}_{B R}, S_{[\tilde{\omega}(t)]}\right)}\left[\mathrm{O}_{R} ;\{\bar{r}\}\right]} \\
& \text { or, equivalently }\left[\mathrm{O}_{B} ;\{b\}\right]_{\mathrm{M}_{L^{\infty}(\Omega, v)}\left(\mathrm{O}_{B R}, S_{[\tilde{\omega}(t)]}\right)}^{\Leftarrow}\left[\mathrm{O}_{R} ;\{r\}\right](\forall t \in U)
\end{aligned}
$$

However, the above term "Let $t$ be any bird" is too logical and not concrete. Thus, we may, by using the parallel measurement $(c f .(\mathrm{H})$ in Definition 8$)$, rewrite the $\left(\mathrm{O}_{1}\right)$ to the following:

$\left(\mathrm{O}_{2}\right)$ Assume that a measured value $\left(x_{t}, y_{t}\right)_{t \in U} \in(X \times Y)^{U}$ is obtained by the parallel measurement $\otimes_{t \in U} \mathrm{M}_{L^{\infty}(\Omega, v)}\left(\mathrm{O}_{B R}:=\left(X \times Y, 2^{X \times Y}, H\right), S_{[\tilde{\omega}(t)]}\right)$. Axiom 1 [measurement] says that the probability that $\left(x_{t}, y_{t}\right) \in\{(b, r),(b, \bar{r}),(\bar{b}, \bar{r})\}$ $(\forall t \in U)$ is equal to 1 .

which is a formal (i.e., measurement theoretical) expression of the $\left(\mathrm{L}_{3}\right)$. Also, as shown in the following [Step(IV)], this $\left(\mathrm{O}_{2}\right)$ is the best compared to $\left(\mathrm{M}_{2}\right)$ and $\left(\mathrm{N}_{2}\right)$.

[Step (IV)]: Answers to Hempel's problems $\left(\mathrm{K}_{3}\right)$ and $\left(\mathrm{K}_{4}\right)$

Although $\left(\mathrm{L}_{1}\right),\left(\mathrm{L}_{2}\right)$ and $\left(\mathrm{L}_{3}\right)$ are equivalent, the measurements $\left(\mathrm{M}_{2}\right),\left(\mathrm{N}_{2}\right)$ and $\left(\mathrm{O}_{2}\right)$ are not equivalent. In $\left(\mathrm{M}_{2}\right)$ of [Step (I)], we consider the parallel measurement $\otimes_{t \in R} \quad \mathrm{M}_{L^{\infty}(\Omega, v)}\left(\mathrm{O}_{B}:=\left(X, 2^{X}, F\right), S_{[\tilde{\omega}(t)]}\right)$, which includes $\#[R]$ measurements. However, in $\left(\mathrm{O}_{2}\right)$ of [Step (III)], we consider the parallel measurement $\otimes_{t \in U} \mathrm{M}_{L^{\infty}(\Omega, v)}\left(\mathrm{O}_{B R}:=\left(X \times Y, 2^{X \times Y}, H\right), S_{[\tilde{\omega}(t)]}\right)$, which includes more measurements than the parallel measurement $\otimes_{t \in R} \mathrm{M}_{L^{\infty}(\Omega, v)}\left(\mathrm{O}_{B}:=\left(X, 2^{X}, F\right), S_{[\tilde{\omega}(t)]}\right)$. Thus, some people think that the actual verification of $\left(\mathrm{L}_{1}\right)$ (or, $\left.\left(\mathrm{L}_{2}\right)\right)$ may be easier than that of $\left(\mathrm{L}_{3}\right)$. However, this is not true. That is because if the $\left(\mathrm{M}_{2}\right)$ asserts "all ravens are black" ( $R \subseteq B$ ), we have to prove that $R$ is the set of all ravens. That is,

$\left(\mathrm{P}_{1}\right)$ Before the measurement $\left(\mathrm{M}_{2}\right)$, we have to prove that $R$ is the set of all ravens, namely, we have to obtain the measured value $\left(y_{t}\right)_{t \in U}$ by the parallel measurement $\otimes_{t \in U} \mathrm{M}_{L^{\infty}(\Omega, v)}\left(\mathrm{O}_{R}:=\left(Y, 2^{Y}, G\right), S_{[\tilde{\omega}(t)]}\right)$, and we define $R$ (the set of all ravens) by $\left\{t \in U \mid y_{t}=r\right\}$. And further, we take the parallel measurement $\otimes_{t \in R} \mathrm{M}_{L^{\infty}(\Omega, v)}\left(\mathrm{O}_{B}:=\left(X, 2^{X}, F\right), S_{[\tilde{\omega}(t)]}\right)$ in $\left(\mathrm{N}_{2}\right)$.

This procedure $\left(\mathrm{P}_{1}\right)$ is realized by the parallel measurement $\otimes_{t \in U} \mathrm{M}_{L^{\infty}(\Omega, v)}\left(\mathrm{O}_{B R}:=\left(X \times Y, 2^{X \times Y}, H\right), S_{[\tilde{\omega}(t)]}\right)$ in $\left(\mathrm{O}_{2}\right)$ of [Step (III)] (if it is 
proved that $U$ is the set of all birds). That is, in any of the three cases $\left(\left(\mathrm{M}_{2}\right),\left(\mathrm{N}_{2}\right)\right.$ and $\left.\left(\mathrm{O}_{2}\right)\right)$, it is discussed under the premise that $U$ is the set of all ravens" is known. Summing up, we conclude that

$\left(\mathrm{P}_{2}\right)$ If $\left(\mathrm{K}_{1}\right)$; " $R \subseteq B(\subseteq U)$ " means "All ravens are black", we have to prove " $R$ is the set of all ravens", not just define it. To do so, we have to examine all the birds $(\forall t \in U)$. That is, we have to prove that $U\left(\equiv\left\{b_{1}, b_{2}, \cdots, b_{\#[U]}\right\}\right)$ is the set of all birds. However, it is usually difficult to prove that $U$ is the set of all birds as there may be some birds in unexplored land. Therefore, in most case, it is impossible to be convinced of "All ravens are black". Similarly, to assert "every non-black bird is a nonraven" or "a non-black raven does not exist", we have to examine all the birds ( $\forall t \in U$ ). This is impossible in most cases.

Therefore, we can completely understand the questions $\left(\mathrm{K}_{3}\right)$ and $\left(\mathrm{K}_{4}\right)$. As the answer to the $\left(\mathrm{K}_{3}\right)$ and $\left(\mathrm{K}_{4}\right)$, some may directly find the $\left(\mathrm{P}_{2}\right)$ without quantum language (and thus, without the arguments [Step(I) Step(IV)]). If so, they may be somewhat excellent. However, it is not worth so much. That is because we think that the reason why Hempel's problem is famous is that many researchers know the $\left(\mathrm{P}_{2}\right)$ (i.e., the difference between definition and proof) unconsciously. Thus, we think that to solve Hempel's raven problems $\left(\mathrm{K}_{3}\right)$ and $\left(\mathrm{K}_{4}\right)$ is to answer the following:

$\left(\mathrm{P}_{3}\right)$ Propose a worldview! And further derive the assertion $\left(\mathrm{P}_{2}\right)$ from the axioms of its worldview!

As shown above $\left[\right.$ Step(I) Step(IV)], we derived the $\left(\mathrm{P}_{2}\right)$ from Axiom 1 [measurement] in the linguistic quantum mechanical worldview.

Remark 17. 1): As seen in the above, the logical implication " $\rightarrow$ " has various interpretation in quantum language. In this paper we are not concerned with Axiom 2 [Causality], which is also related to "implication". That is because ["state at time $t_{1}$ " $\frac{t_{1}<t_{2}}{\text { Axiom } 2 \text { causality] }}$ "state at time $t_{2}$ "] can be regarded as a kind of implication. But it's a little unreasonable to regard causality as an implication. For example, consider the following famous puzzle:

$\left(\mathrm{P}_{4}\right)$ Describe the contraposition of "If he is not scolded, he does not study"!

This is not so difficult as puzzle. Also, some may associate temporal logic. But, we think, from the quantum linguistic point of view, that this puzzle is unnatural. That is because we consider that the language of science is not logic but quantum language.

2): For the sake of completeness, we sum up and add the following correspondence:

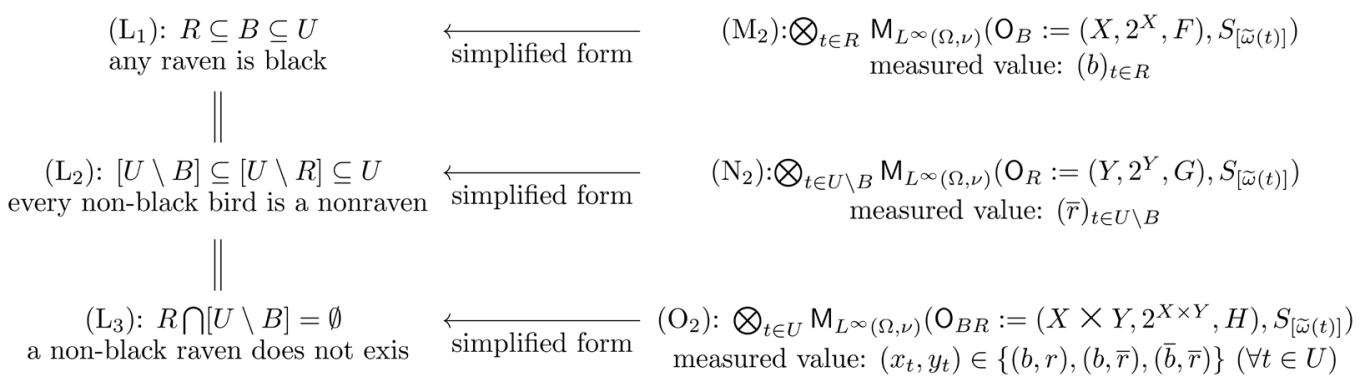
$\left(\mathrm{L}_{2}\right):[U \backslash B] \subseteq[U \backslash R] \subseteq U$
every non-black bird is a nonraven
simplified form

$\overleftarrow{\text { simplified form }}$ $\overleftarrow{\text { simplified form }}$ 


\subsection{Falsification Test}

As seen in the above $\left(\mathrm{P}_{2}\right)$, in most case, it is impossible to be convinced of "All ravens are black". Thus, our next problem is to answer the problem "How do we believe it?". For example, assume the following fact:

$\left(Q_{1}\right)$ It was found that one hundred ravens were black continuously.

What we can do is to reject the null hypothesis of the $\left(\mathrm{Q}_{1}\right)$. For instance, it is usual to assume the following null hypothesis:

$\left(Q_{2}\right)$ Non-black ravens can be observed at 3 time of a rate to 100 times.

A simple calculation shows that this null hypothesis $\left(Q_{2}\right)$ is represented in quantum language as the measurement $\mathrm{M}_{L^{\infty}(\Omega, v)}\left(\mathrm{O}_{B}^{\prime}:=\left(X, 2^{X}, F^{\prime}\right), S_{[\tilde{\omega}(r)]}\right)$, (where $\tilde{\omega}: U \rightarrow \Omega$ is the state map (cf. the formula (3)) such that $\tilde{\omega}(r) \in \Omega_{R}^{\circ}$ $(\forall r \in R))$ where

$$
\begin{aligned}
& X=\{b, \bar{b}\}, \\
& {\left[F^{\prime}(\{b\})\right](\omega)=\frac{97}{100} \quad\left(\text { if } \omega \in \Omega_{R}\right), 0 \leq\left[F^{\prime}(\{b\})\right](\omega) \leq 1 \quad\left(\text { if } \omega \in \Omega \backslash \Omega_{R}\right),} \\
& {\left[F^{\prime}(\{\bar{b}\})\right](\omega)=1-\left[F^{\prime}(\{b\})\right](\omega)(\forall \omega \in \Omega)}
\end{aligned}
$$

which is a slight modification of the formula (4). And thus, under the null hypothesis $\left(\mathrm{Q}_{2}\right)$, we calculate, by Axiom 1 [measurement], that

$\left(\mathrm{Q}_{3}\right)$ the probability that a measured value $\left(x_{i}\right)_{i=1}^{100}\left(\in X^{100}\right)$ obtained by the parallel measurement

$\otimes_{r \in\left\{r_{1}, r_{2}, \cdots, r_{100}\right\} \subseteq R} \mathrm{M}_{L^{\infty}(\Omega, v)}\left(\mathrm{O}_{B}^{\prime}:=\left(X, 2^{X}, F^{\prime}\right), S_{[\tilde{\omega}(r)]}\right)$ satisfies that $x_{i}=b(i=1,2, \cdots, 100)$ is given by $(97 / 100)^{100}(<0.048)$. That is, the probability that $\left(\mathrm{Q}_{1}\right)$ is realized (i.e., we meet one hundred black ravens continuously) is less than $\left.0.048\left(>(97 / 100)^{100}\right)\right)$.

Thus, we may reject the null hypothesis $\left(\mathrm{Q}_{2}\right)$ since probability 0.048 is quite rare.

Remark 18. Note that the above argument is popular as statistical hypothesis testing in statistics, though statistics does not have the concept of "measurement". In our worldview (i.e., linguistic quantum mechanical worldview), we consider that Popper's falsificationism (cf, ref. [27]) and statistical hypothesis testing are almost the same. However his theory was supported by philosophers rather than scientists since his proposal was not proposed under a certain scientific worldview. That is, Popper's falsificationism belongs to $\left(A_{1}\right)$ : "philosophy of science for the general public".

\section{Hume's Problem of Induction}

\subsection{Problem of Induction in the Linguistic Quantum Mechanical World View}

Although David Hume (1711-1776), British experimentalist, suspected the justification of induction, in this section we show that the justification is easily solved in our worldview. If we expect a scientific answer to Hume's problem, we must 
start with the scientific definition of "the uniformity principle of nature", i.e., the following Definition 19 [The uniformity principle of nature]. Some may feel that the uniformity principle of nature (i.e., the condition in Definition 19) is too strong. However, we think that it is impossible to propose the different quantitative definition of the uniformity principle of nature that leads to a result like Theorem 20 [Inductive reasoning] (i.e., If similar measurements are performed, the similar measured values are obtained).

Definition 19. [The uniformity principle of nature] Let $\left[C(\Omega) \subseteq L^{\infty}(\Omega, v) \subseteq B\left(L^{2}(\Omega, v)\right)\right]$ be a classical basic structure such that $\Omega$ is compact and $v(\Omega)=1$. A family of measurements $\left\{\mathrm{M}_{L^{\infty}(\Omega, v)}\left(\mathrm{O}_{i}:=\left(X, \mathcal{F}, F_{i}\right), S_{\left[\omega_{i}\right]}\right) \mid i=-n,-n+1, \cdots,-1,0,1,2, \cdots, N\right\}$ is said to satisfy the uniformity principle of nature (concerning $\mu$ ), if there exists a probability space $(X, \mathcal{F}, \mu)$ such that

$$
\left[F_{i}(\Xi)\right]\left(\omega_{i}\right)=\mu(\Xi) \quad \forall \Xi \in \mathcal{F}, \forall i=-n,-n+1, \cdots,-1,0,1,2, \cdots, N
$$

Under this definition, we assert the following theorem, which should be regarded as the fundamental theorem in philosophy of science.

Theorem 20. [Inductive reasoning, the quantum linguistic solution of Hume's problem of induction]. Let $\left[C(\Omega) \subseteq L^{\infty}(\Omega, v) \subseteq B\left(L^{2}(\Omega, v)\right)\right]$ be a basic structure such that $\Omega$ is compact and $v(\Omega)=1$. Assume that a family of measurements

$\left\{\mathrm{M}_{L^{\infty}(\Omega, v)}\left(\mathrm{O}_{i}:=\left(X, \mathcal{F}, F_{i}\right), S_{\left[\omega_{i}\right]}\right) \mid i=-n,-n+1, \cdots,-1,0,1,2, \cdots, N\right\}$ satisfies the uniformity principle of nature (concerning $\mu$ ). Let $\left(x_{-n}, x_{-n+1}, \cdots, x_{-1}, x_{0}, x_{1}, \cdots, x_{N}\right) \in \times_{i=-n}^{N} X$ be a measured value by the parallel measurement $\otimes_{i=-n}^{N} \mathrm{M}_{L^{\infty}(\Omega, v)}\left(\mathrm{O}_{i}:=\left(X, \mathcal{F}, F_{i}\right), S_{\left[\omega_{i}\right]}\right)$. Then, we see that

$$
\begin{aligned}
& \frac{\#\left\{k \mid x_{k} \in \Xi, k=-n,-n+1, \cdots,-1,0\right\}}{n} \\
& \approx \mu(\Xi)\left(=\left[F_{i}(\Xi)\right]\left(\omega_{i}\right)\right) \quad(\Xi \in \mathcal{F}, i=-n,-n+1, \cdots,-1,0,1,2, \cdots, N)
\end{aligned}
$$

where $n$ is sufficiently large. Here $\#[\Theta]$ is the number of elements in a set $\Theta$.

Proof. Let $\Xi_{i} \in \mathcal{F} \quad(i=-n,-n+1, \cdots,-1,0,1, \cdots, N)$. Axiom 1 [measurement] says that the probability that a measured value $\left(x_{-n}, x_{-n+1}, \cdots, x_{-1}, x_{0}, x_{1}, \cdots, x_{N}\right)$ obtained by the parallel measurement $\otimes_{i=-n}^{N} \mathrm{M}_{L^{\infty}(\Omega, v)}\left(\mathrm{O}_{i}:=\left(X, \mathcal{F}, F_{i}\right), S_{\left[\omega_{i}\right]}\right)$ belongs to $\times_{i=-n}^{N} \Xi_{i}$ is given by $\times_{i=-n}^{N}\left[F_{i}\left(\Xi_{i}\right)\right]\left(\omega_{i}\right)=\times_{i=-n}^{N} \mu\left(\Xi_{i}\right)$. Thus, the sequence $\left\{x_{i}\right\}_{i=-n}^{N}$ can be regarded as independent random variables with the identical distribution $\mu$. Hence, using the law of large numbers, we can immediately get the formula (7). Also, this theorem is a direct consequence of the law of large numbers for parallel measurements ( $c f$. refs. [5], or $\$ 4.2$ in ref. [18]).

Remark 21. 1): Recall that the law of large numbers (which is almost equivalent to Theorem 20) says that "frequency probability" = "the probability in Axiom 1" (cf. ref. [5]) though the probability in Axiom 1 has the several aspects. 
Also, note that the law of large numbers in statistics (cf. ref. [26]) has already been accepted as the fundamental theorem in science. Therefore, even if Theorem 20 ([Inductive reasoning] + (7)) is called the fundamental theorem in philosophy of science, we don't think it's exaggerated. We believe that our proposal (i.e., Theorem 20) is completely true in our worldview. Thus, we think that the solution of Hume's problem of induction was practically already found as the law of large numbers. In the framework of our worldview, we are convinced that the above is the definitive solution to Hume's problem. However, there may be another idea if some start from another worldview. Hence, as described at the end of this paper, we hope that many philosophers propose various mathematical foundations of scientific philosophy, in which Hume's problem of induction are discussed from the various viewpoints.

2): In Definition 19 [The uniformity principle of nature] and Theorem 20 [Inductive reasoning], we consider the family of measurements $\left\{\mathrm{M}_{L^{\infty}(\Omega, v)}\left(\mathrm{O}_{i}:=\left(X, \mathcal{F}, F_{i}\right), S_{\left[\omega_{i}\right]}\right) \mid i=-n,-n+1, \cdots,-1,0,1,2, \cdots, N\right\}$. This may be too general. Usually, it suffices to consider that

$\left\{\mathrm{M}_{L^{\infty}(\Omega, v)}\left(\mathrm{O}_{i}:=\left(X, \mathcal{F}, F_{i}\right), S_{\left[\omega_{i}\right]}\right) \mid i=-n,-n+1, \cdots,-1,0,1,2, \cdots, N\right\}$, i.e., $\quad F=F_{i}$ $(-n \leq \forall i \leq N)$.

3): It may be understandable to consider two measurements:

$\otimes_{i=-n}^{0} \mathrm{M}_{L^{\infty}(\Omega, v)}\left(\mathrm{O}:=\left(X, \mathcal{F}, F_{i}\right), S_{\left[\omega_{i}\right]}\right) \quad$ and $\otimes_{i=1}^{N} \mathrm{M}_{L^{\infty}(\Omega, v)}\left(\mathrm{O}:=\left(X, \mathcal{F}, F_{i}\right), S_{\left[\omega_{i}\right]}\right)$.

The reason that we do not consider two measurements is due to the linguistic Copenhagen interpretation $\left(\mathrm{G}_{1}\right)$, i.e., only one measurement is permitted.

Example 22. [Coin tossing]. Let us discuss the unfair coin tossing as the most understandable example of Theorem 20 [Inductive reasoning]. Consider a basic structure $\left[C(\Omega) \subseteq L^{\infty}(\Omega, v) \subseteq B\left(L^{2}(\Omega, v)\right)\right]$. Let $\left\{\omega_{i}\right\}_{i=-n}^{N}$ be a sequence in $\Omega$, where $\omega_{i}$ is the state of $i$-th coin tossing $(i=-n,-n+1, \cdots, 0,1,2,3, \cdots, N)$. Let $\mathrm{O}=\left(X, 2^{X}, F\right)$ be an observable in $L^{\infty}(\Omega, v)$ such that

$$
\begin{aligned}
X & =\{H, T\},(\text { where } H: \text { head, } U: \text { tail }), \\
& {[F(\{H\})]\left(\omega_{i}\right)=\mu(\{H\})=2 / 3, } \\
& {[F(\{T\})]\left(\omega_{i}\right)=\mu(\{T\})=1 / 3 \quad(\forall i=-n,-n+1, \cdots,-1,0,1,2, \cdots, N) }
\end{aligned}
$$

That is, a family of measurements $\left\{\mathrm{M}_{L^{\infty}(\Omega, v)}\left(\mathrm{O}:=\left(X, 2^{X}, F\right), S_{\left[\omega_{i}\right]}\right) \mid i=-n,-n+1, \cdots,-1,0,1,2, \cdots, N\right\}$ satisfies the uniformity principle of nature (concerning $\mu$ ). Let

$\left(x_{-n}, x_{-n+1}, \cdots, x_{-1}, x_{0}, x_{1}, \cdots, x_{N}\right) \in x_{i=-n}^{N} X$ be a measured value obtained by the parallel measurement $\otimes_{i=-n}^{N} \mathrm{M}_{L^{\infty}(\Omega, v)}\left(\mathrm{O}:=\left(X, 2^{X}, F\right), S_{\left[\omega_{i}\right]}\right)$, i.e., infinite coin throws. Here, Theorem 20 [Inductive reasoning] say that it is natural to assume that, for sufficiently large $n$,

$$
\left(x_{-n}, x_{-n+1}, \cdots, x_{-1}, x_{0}\right)=(\underbrace{T H H T H H H T T \cdots T H H}_{n+1})
$$

(where the number of $H \mathrm{~s} \approx 2 n / 3, T \mathrm{~s} \approx n / 3$ ) 
Then we can believe that we see that $x_{i}=H$ with probability 2/3 [resp. $x_{i}=T$ with probability $1 / 3$ ] for each $i=1,2, \cdots, N$. It should be noted that even without knowing (8), we can conclude that if we know (9).

Remark 23. It should be noted that the above example shows that Theorem 20 [Inductive reasoning] (or equivalently, the law of large numbers), like Newton's kinetic equation, has the power to predict the future. This is the reason that Hume's problem of induction keeps attracting much researcher's interest for a long time.

Example 24. [Induction concerning raven problem]. Let $R \subseteq B \subseteq U$ be as in Section 3.2. Consider a basic structure $\left[C(\Omega) \subseteq L^{\infty}(\Omega, v) \subseteq B\left(L^{2}(\Omega, v)\right)\right]$. Let $\mathrm{O}_{B}=\left(X, 2^{X}, F\right)$ be an observable in $L^{\infty}(\Omega, v)$ such as defined in the formula (4), that is,

$$
\begin{aligned}
& X=\{b, \bar{b}\}, \\
& \left.[F(\{b\})](\omega)=1 \quad\left(\text { if } \omega \in \Omega_{B}\right),[F(\{b\})](\omega)=0 \quad \text { (if } \omega \in \Omega \backslash \Omega_{B}\right), \\
& {[F(\{\bar{b}\})](\omega)=1-[F(\{b\})](\omega)(\forall \omega \in \Omega)}
\end{aligned}
$$

Let $\left\{\omega_{i}\right\}_{i=-n}^{N}$ be a sequence in $\Omega_{R}^{\circ}(\subseteq \Omega)$. Clearly, a family of measurements $\left\{\mathrm{M}_{L^{\infty}(\Omega, v)}\left(\mathrm{O}_{B}:=\left(X, 2^{X}, F\right), S_{\left[\omega_{i}\right]}\right) \mid i=-n,-n+1, \cdots,-1,0,1,2, \cdots, N\right\}$ satisfies the uniformity principle of nature (concerning $\mu$ ) such that

$$
\begin{aligned}
& {[F(\{b\})]\left(\omega_{i}\right)=\mu(\{b\})=1,} \\
& {[F(\{\bar{b}\})]\left(\omega_{i}\right)=\mu(\{\bar{b}\})=0, \quad(i=-n,-n+1, \cdots, 0,1,2,3, \cdots, N)}
\end{aligned}
$$

Let $\left(x_{-n}, x_{-n+1}, \cdots, x_{-1}, x_{0}, x_{1}, \cdots, x_{N}\right) \in \times_{i=-n}^{N} X$ be a measured value obtained by the parallel measurement $\otimes_{i=-n}^{N} \mathrm{M}_{L^{\infty}(\Omega, v)}\left(\mathrm{O}_{B}:=\left(X, 2^{X}, F\right), S_{\left[\omega_{i}\right]}\right)$, We see, of course, that $x_{i}=b \quad(i=-n,-n+1, \cdots,-1,0)$. And thus, we can believe, by Theorem 20 [Inductive reasoning], that $x_{1}=x_{2}=\cdots=x_{N}=b$.

\subsection{Grue Paradox Cannot Be Represented in Quantum Language}

If our understanding of inductive reasoning (mentioned in the above) is true, we can solve the grue paradox ( $c f$. ref. [28]). Let us mention it as follows.

Consider a basic structure $\left[C(\Omega) \subseteq L^{\infty}(\Omega, v) \subseteq B\left(L^{2}(\Omega, v)\right)\right]$. Let $\Omega_{g}, \Omega_{b}, \Omega_{o}$ be the subsets of the state space $\Omega$ such that $\Omega_{g} \cap \Omega_{b}=\varnothing$ and $\Omega_{o}=\Omega \backslash\left(\Omega_{g} \cup \Omega_{b}\right)$. Let $\mathrm{O}=\left(X \equiv\{g, b, o\}, 2^{X}, F\right)$ be the observable in $L^{\infty}(\Omega, v)$ such that

$$
\begin{aligned}
& {[F(\{(g)\})](\omega)=1\left(\omega \in \Omega_{g}\right), \quad=0\left(\omega \in \Omega \backslash \Omega_{g}\right)} \\
& {[F(\{(b)\})](\omega)=1\left(\omega \in \Omega_{b}\right), \quad=0 \quad\left(\omega \in \Omega \backslash \Omega_{b}\right)} \\
& {[F(\{(o)\})](\omega)=1-[F(\{(g)\})](\omega)-[F(\{(b)\})](\omega)(\omega \in \Omega)}
\end{aligned}
$$

where " $g$ ", “ $b$ ", " $o$ " respectively means "green", "blue", "others".

Let $\left\{e_{-n}, e_{-n+1}, \cdots, e_{-1}, e_{0}, e_{1}, e_{2}, \cdots, e_{N}\right\}$ be the set of (green) emeralds. And as- 
sume that $\omega_{i}\left(\in \Omega_{g}^{\circ}\right)$ is the state of emerald $e_{i}$ $(i=-n,-n+1, \cdots,-1,0,1,2, \cdots, N)$.

A family of measurements

$\left\{\mathrm{M}_{L^{\infty}(\Omega, v)}\left(\mathrm{O}_{i}:=\left(X, 2^{X}, F\right), S_{\left[\omega_{i}\right]}\right) \mid i=-n,-n+1, \cdots,-1,0,1,2, \cdots, N\right\} \quad$ clearly satisfies the uniformity principle of nature, that is, there exists an probability space $\left(X, 2^{X}, \mu\right)$ such that

$$
[F(\Xi)]\left(\omega_{i}\right)=\mu(\Xi) \quad \forall \Xi \in 2^{X}, \forall i=-n,-n+1, \cdots,-1,0,1,2, \cdots, N
$$

where $\mu(\{g\})=1, \mu(\{b, o\})=0$.

Let $\left(x_{-n}, x_{-n+1}, \cdots, x_{-1}, x_{0}, x_{1}, \cdots, x_{N}\right) \in \times_{i=-n}^{N} X$ be a measured value obtained by the parallel measurement $\otimes_{i=-n}^{N} \mathrm{M}_{L^{\infty}(\Omega, v)}\left(\mathrm{O}:=\left(X, 2^{X}, F\right), S_{\left[\omega_{i}\right]}\right)$. We see, of course, that $x_{i}=g(i=-n,-n+1, \cdots,-1,0)$. And thus, we can believe, by Theorem 20 [Inductive reasoning], that $x_{1}=x_{2}=\cdots=x_{N}=g$. For the sake of completeness, note that we can predict $x_{1}=x_{2}=\cdots=x_{N}=g$ only by the data $x_{-n}=x_{-n+1}=\cdots=x_{0}=g$. This is usual arguments concerning Theorem 20 [Inductive reasoning].

On the other hand, Goodman's grue paradox is as follows (cf. ref. [28]).

$\left(\mathrm{R}_{1}\right)$ Define that $Y$ has a grue property iff $Y$ is green at time $i$ such that $i \leq 0$ and $Y$ is blue at time $i$ such that $0<i$. Suppose that we have examined the emeralds at $-n,-n+1, \cdots,-1,0$, and found them to all be green (and hence also grue). Then, "so-called inductive reasoning" says that emeralds at $1,2, \cdots, N$ have the grue property (and hence blue) as well as green. Thus, a contradiction is gotten.

However, we think that this $\left(R_{1}\right)$ cannot be described in quantum language. If we try to describe the $\left(\mathrm{R}_{1}\right)$, we may consider as follows.

$\left(\mathrm{R}_{2}\right)$ Let $\left\{e_{-n}, e_{-n+1}, \cdots, e_{-1}, e_{0}, e_{1}, e_{2}, \cdots, e_{N}\right\}$ be the set of emeralds. Let $\omega_{i}\left(\in \Omega_{g}^{\circ}\right)$ be the state of emerald $e_{i}(i=-n,-n+1, \cdots,-1,0)$, and let $\omega_{i}\left(\in \Omega_{b}^{\circ}\right)$ be the state of emerald $e_{i} \quad(i=1,2, \cdots, N)$. However, it should be noted that a family of measurements

$\left\{\mathrm{M}_{L^{\infty}(\Omega, v)}\left(\mathrm{O}_{i}:=\left(X, 2^{X}, F_{i}\right), S_{\left[\omega_{i}\right]}\right) \mid i=-n,-n+1, \cdots,-1,0,1,2, \cdots, N\right\}$ does not satisfy the uniformity principle of nature. That is because

$$
\begin{aligned}
& {[F(\{g\})]\left(\omega_{i}\right)=1(i=-n,-n+1, \cdots, 0),} \\
& {[F(\{g\})]\left(\omega_{i}\right)=0(i=1,2, \cdots, N)}
\end{aligned}
$$

Hence Theorem 20 [Inductive reasoning] cannot be applied.

Or,

$\left(\mathrm{R}_{3}\right)$ Let $\left\{e_{-n}, e_{-n+1}, \cdots, e_{-1}, e_{0}, e_{1}, e_{2}, \cdots, e_{N}\right\}$ be the set of emeralds. And let $\omega_{i}\left(\in \Omega_{g}^{\circ}\right)$ is the state of emerald $e_{i}$ such that $\omega=\omega_{i}$ $(i=-n,-n+1, \cdots,-1,0,1,2, \cdots, N)$. Let $\mathrm{O}_{i}=\left(X, 2^{X}, F_{i}\right)$ be the observable $(i=-n,-n+1, \cdots,-1,0,1,2, \cdots, N)$ such that $\mathrm{O}_{i}$ is the same as $\mathrm{O}\left(=\left(X \equiv\{g, b, o\}, 2^{X}, F\right)\right)$ in (10) (if $\left.i=-n,-n+1, \cdots,-1,0\right)$, and $\mathrm{O}_{i}=\left(X, 2^{X}, F_{i}\right)$ (if $\left.0,1,2, \cdots, N\right)$ is defined by $F_{i}(\{g\})=F(\{b\})$, $F_{i}(\{b\})=F(\{g\}), F_{i}(\{o\})=F(\{o\})$. However, in this case, it should be noted 
that a family of measurements

$\left\{\mathrm{M}_{L^{\infty}(\Omega, v)}\left(\mathrm{O}_{i}:=\left(X, 2^{X}, F_{i}\right), S_{\left[\omega_{i}\right]}\right) \mid i=-n,-n+1, \cdots,-1,0,1,2, \cdots, N\right\}$ does not sa-

tisfy the uniformity principle of nature. That is because

$$
\begin{aligned}
& {\left[F_{i}(\{g\})\right]\left(\omega_{i}\right)=[F(\{g\})]\left(\omega_{i}\right)=1 \quad(i=-n,-n+1, \cdots, 0),} \\
& {\left[F_{i}(\{g\})\right]\left(\omega_{i}\right)=[F(\{b\})]\left(\omega_{i}\right)=0 \quad(i=1,2, \cdots, N)}
\end{aligned}
$$

Hence Theorem 20 [Inductive reasoning] cannot be applied.

Therefore Goodman's grue paradox $\left(R_{1}\right)$ cannot be described in quantum language.

Remark 25. We believe that there is no scientific argument without scientific worldview. Thus, we can immediately conclude that Goodman's discussion $\left(\mathrm{R}_{1}\right)$ is doubtful since his argument is not based on any scientific worldview. In this sense, the above arguments $\left(R_{2}\right)$ and $\left(R_{3}\right)$ may not be needed. That is, the confusion of grue paradox is due to lack of the understanding of Hume's problem of induction in the linguistic quantum mechanical worldview, and not lack of the term "grue" is non-projectible ( $c f$. ref. [28]). Thus, we think that to solve Goodman's grue paradox is to answer the following:

$\left(\mathrm{R}_{4}\right)$ Propose a worldview! And further formulate Hume's induction as the fundamental theorem in the worldview! In this formulation, confirm that Goodman's paradox is eliminated naturally.

What we did is this.

\section{The Measurement Theoretical Representation of Abduction}

\subsection{Deduction and Abduction in "Logic"}

A typical example of deduction is as follows: (In the following, $\left(\mathrm{S}_{1}^{\prime}\right)$ and $\left(\mathrm{T}_{1}^{\prime}\right)$ are often omitted.)

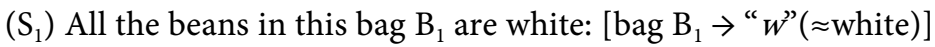

$\left(S_{2}\right)$ All the beans in that bag $B_{2}$ are white or black fifty-fifty (or generally, the ratio of white beans to black beans is $p /(1-p)$ where $0<p<1)$ : [bag $\mathrm{B}_{2} \rightarrow$

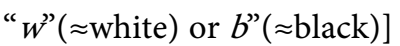

$\left(\mathrm{S}_{3}\right)$ This bean is from this bag $\mathrm{B}_{1}$ : $\left[\right.$ bag $\left.\mathrm{B}_{1}\right]$

$\left(\mathrm{S}_{4}\right)$ Therefore, this bean is white: [“" $w^{\text {" ( }} \approx$ white $\left.)\right]$

It is, of course, obvious and ordinary.

On the other hand, C.S, Peirce (cf. ref. [29]) proposed abduction. The example of abduction is as follows:

$\left(\mathrm{T}_{1}\right)$ All the beans in this bag $\mathrm{B}_{1}$ are white: $\left[\right.$ bag $\mathrm{B}_{1} \rightarrow$ "w" $(\approx$ white $\left.)\right]$

$\left(\mathrm{T}_{1}^{\prime}\right)$ All the beans in that bag $\mathrm{B}_{2}$ are white and black fifty-fifty (or generally, the ratio of white beans to black beans is $p /(1-p)$ ): [bag $\mathrm{B}_{2} \rightarrow$ "w" ( $\approx$ white) or

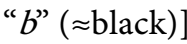

$\left(\mathrm{T}_{2}\right)$ This bean (from $\mathrm{B}_{1}$ or $\mathrm{B}_{2}$ ) is white: [“w”( $\approx$ white)]

$\left(T_{3}\right)$ Therefore, this bean is from this bag $B_{1}$ : [bag $\left.B_{1}\right]$ 
This is wrong from the logical point of view. However, the abduction (abductive reasoning) is known as one of useful tools to find a best solution. Also, note that $\left[\left(\mathrm{S}_{2}\right) \rightarrow\left(\mathrm{S}_{3}\right)\right]$ and $\left[\left(\mathrm{T}_{2}\right) \rightarrow\left(\mathrm{T}_{3}\right)\right]$ are in reverse relation.

\subsection{The Measurement Theoretical Representation of Deduction and Abduction}

In this section, we show that the abduction $\left[\left(\mathrm{T}_{1}\right)-\left(\mathrm{T}_{3}\right)\right]$ can be justified in quantum language. Consider the state space $\Theta=\left\{\theta_{1}, \theta_{2}\right\}$ with the discrete topology, and the classical basic structure $\left[C(\Theta) \subseteq L^{\infty}(\Theta, v) \subseteq B\left(L^{2}(\Theta, v)\right)\right]$, where $v\left(\left\{\theta_{1}\right\}\right)=v\left(\left\{\theta_{2}\right\}\right)=1 / 2$. Assume that

$$
\theta_{1} \approx \text { the state of the bag } \mathrm{B}_{1}, \quad \theta_{2} \approx \text { the state of the bag } \mathrm{B}_{2},
$$

Assume that 1000 white beans belong to bag $B_{1}$, and further, 500 white beans and 500 black beans belong to the bag $\mathrm{B}_{2}$. Thus we have the observable $\mathrm{O}=\left(\{w, b\}, 2^{\{w, b\}}, F\right)$ in $L^{\infty}(\Theta, v)$ such that

$$
\begin{gathered}
{[F(\{w\})]\left(\theta_{1}\right)=1 \quad[F(\{b\})]\left(\theta_{1}\right)=0} \\
{[F(\{w\})]\left(\theta_{2}\right)=p \quad[F(\{b\})]\left(\theta_{2}\right)=1-p \quad(0<p<1)}
\end{gathered}
$$

where " $w$ " and " $b$ " means "white" and "black" respectively.

Thus, we have the measurement $\mathrm{M}_{L^{\infty}(\Theta, v)}\left(\mathrm{O}:=\left(\{w, b\}, 2^{\{w, b\}}, F\right), S_{\left[\theta_{i}\right]}\right)$, $i=1,2$. For example, Axiom 1 [measurement] says that

$\left(\mathrm{U}_{1}\right)$ [measurement]: The probability that the measured value $w$ is obtained by $\mathrm{M}_{L^{\infty}(\Theta, v)}\left(\mathrm{O}:=\left(\{w, b\}, 2^{\{w, b\}}, F\right), S_{\left[\theta_{1}\right]}\right)$ is equal to 1

This is the same as the deduction (i.e., $\left(\mathrm{S}_{1}\right)-\left(\mathrm{S}_{3}\right)$ ).

Next, under the circumstance that bags $B_{1}$ and $B_{2}$ cannot be distinguished, we consider the following inference problem:

$\left(\mathrm{U}_{2}\right)$ [inference problem]: When the measured value $w$ is obtained by the measurement $\mathrm{M}_{L^{\infty}(\Theta, v)}\left(\mathrm{O}:=\left(\{w, b\}, 2^{\{w, b\}}, F\right), S_{[*]}\right)$, which do you infer, $[*]=\theta_{1}$ or $[*]=\theta_{2}$ ?

Theorem 13 [Fisher's maximum likelihood method] says that $[*]=\theta_{1}$, since

$$
\max \left\{[F(\{w\})]\left(\theta_{1}\right), F[F(\{w\})]\left(\theta_{2}\right)\right\}=\max \{1, p\}=1=[F(\{w\})]\left(\theta_{1}\right)
$$

This implies $\left(\mathrm{T}_{3}\right)$.

Therefore, the above $\left(\mathrm{U}_{2}\right)$ is the measurement theoretical representation of abduction (i.e., $\left.\left(\mathrm{T}_{1}\right)-\left(\mathrm{T}_{3}\right)\right)$. For the sake of completeness, note that $\left(\mathrm{U}_{1}\right)$ and $\left(\mathrm{U}_{2}\right)$ are in reverse problem (cf. Remark 14). That is, we have the following correspondence:

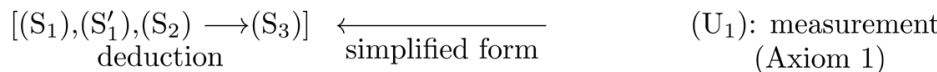

$$
\begin{aligned}
& \text { reverse } \quad \text { reverse } \\
& {\left[\left(\mathrm{T}_{1}\right),\left(\mathrm{T}_{1}^{\prime}\right),\left(\mathrm{T}_{2}\right) \longrightarrow\left(\mathrm{T}_{3}\right)\right] \longleftarrow \quad\left(\mathrm{U}_{2}\right) \text { : inference }} \\
& \text { abduction simplified form (Fisher's maximum likelihood method) }
\end{aligned}
$$

Thus, the scientific meaning of abduction can be completely clarified in the translation from logic to quantum language. 


\section{Flagpole Problem}

Let us explain the flagpole problem as follows. Suppose that the sun is at an elevation angle $\alpha^{\circ}$ in the sky. Assume that $\tan \alpha^{\circ}=1 / 2$. There is a flagpole which is $\omega_{0}^{0}$ meters tall. The flagpole casts a shadow $\omega_{1}^{0}$ meters long. Suppose that we want to explain the length of the flagpole's shadow. On Hempel's model, the following explanation is sufficient.

$\left.\left(V_{1}\right) 1\right)$ The sun is at an elevation angle $\alpha^{\circ}$ in the sky.

2) Light propagates linearly.

3) The flagpole is $\omega_{0}^{0}$ meters high.

Then,

4) The length of the shadow is $\omega_{1}^{0}=\omega_{0}^{0} / \tan \alpha^{\circ}=2 \omega_{0}^{0}$

This is a good explanation of "Why is that shadow $2 \omega_{0}^{0}$ meters long?"

Similarly, we may consider as follows.

$\left(V_{2}\right)$ 1) The sun is at an elevation angle $\alpha^{\circ}$ in the sky.

2) Light propagates linearly.

3) The length of the shadow is $\omega_{1}^{0}$.

Then,

4) The flagpole is $\omega_{0}^{0}\left(=\left(\tan \alpha^{\circ}\right) \omega_{1}^{0}=\omega_{1}^{0} / 2\right)$ meters tall.

However, this is not sufficient as the explanation of "Why is the flagpole $\omega_{0}^{0}\left(=\omega_{1}^{0} / 2\right)$ meters tall?"

The confusion between $\left(\mathrm{V}_{1}\right)$ and $\left(\mathrm{V}_{2}\right)$ is due to the lack of measurement. In what follows, we discuss it. For each time $t=0,1$, consider a basic structure $\left[C\left(\Omega_{t}\right) \subseteq L^{\infty}\left(\Omega_{t}, v_{t}\right) \subseteq B\left(L^{2}\left(\Omega_{t}, v_{t}\right)\right)\right]$, where $\Omega_{0}=[0,1]$ is the state space (in which the length of the flagpole is represented) at time 0 (where the closed interval in the real line $\mathbb{R}$ ), $\Omega_{1}=[0,2]$ is the state space (in which the length of the shadow is represented) at time 1 and the $v_{t}$ is the Lebesgue measure. Since the sun is at an elevation angle $\alpha^{\circ}$ in the sky, it suffices to consider to the map $\phi_{0,1}: \Omega_{0} \rightarrow \Omega_{1}$ such that $\phi_{0,1}\left(\omega_{0}\right)=2 \omega_{0}\left(\forall \omega_{0} \in \Omega_{0}\right)$. Thus, we can define the causal operator $\Phi_{0,1}: L^{\infty}\left(\Omega_{1}\right) \rightarrow L^{\infty}\left(\Omega_{0}\right)$ such that $\left(\Phi_{0,1} f_{1}\right)\left(\omega_{0}\right)=f_{1}\left(\phi\left(\omega_{0}\right)\right)\left(\forall f_{1} \in L^{\infty}\left(\Omega_{1}\right), \omega_{0} \in \Omega_{0}\right)$.

Let $\mathrm{O}_{e}=\left(X, \mathcal{F}, F_{e}\right)$ be the exact observable in $L^{\infty}\left(\Omega_{1}, v_{1}\right)$ (cf. Example 5). That is, it satisfies that $X=\Omega_{1}, \mathcal{F}=\mathcal{B}_{\Omega_{1}}$ (i.e., the Borel field in $\Omega$ ), $\left[F_{e}(\Xi)\right]\left(\omega_{1}\right)=1 \quad$ (if $\left.\omega_{1} \in \Xi\right),=0$ (otherwise).

Thus, we have the measurement $\mathrm{M}_{L^{\infty}\left(\Omega_{0}, v_{0}\right)}\left(\Phi_{0,1} \mathrm{O}_{e}=\left(X, \mathcal{F}, \Phi_{0,1} F_{e}\right), S_{\left[\omega_{0}^{0}\right]}\right)$. Then we have the following statement

$\left(\mathrm{W}_{1}\right)$ [Measurement]; the probability that the measured value $x(\in X)$ obtained by the measurement $\mathrm{M}_{L^{\infty}\left(\Omega_{0}, \nu_{0}\right)}\left(\Phi_{0,1} \mathrm{O}_{e}=\left(X, \mathcal{F}, \Phi_{0,1} F_{e}\right), S_{\left[\omega_{0}^{0}\right]}\right)$ is equal to $2 \omega_{0}^{0}$ is given by 1 .

which is the measurement theoretical representation of $\left(\mathrm{V}_{1}\right)$. That is, we consider that the $\left(\mathrm{V}_{1}\right)$ is the simplified form (or, the rough representation) of $\left(\mathrm{W}_{1}\right)$. Also,

$\left(\mathrm{W}_{2}\right)$ [Inference]; Assume that the measured value $\omega_{1}^{0}(\in X)$ is obtained by the measurement $\mathrm{M}_{L^{\infty}\left(\Omega_{0}, \nu_{0}\right)}\left(\Phi_{0,1} \mathrm{O}_{e}=\left(X, \mathcal{F}, \Phi_{0,1} F_{e}\right), S_{[*]}\right)$. Then, we can infer 
that $[*]=\omega_{1}^{0} / 2$

which is the measurement theoretical representation of $\left(\mathrm{V}_{2}\right)$. That is, we consider that the $\left(\mathrm{V}_{2}\right)$ is the simplified form (or, the rough representation) of $\left(\mathrm{W}_{2}\right)$.

Thus, we conclude that "scientific explanation" is to describe by quantum language. Also, we have to add that the flagpole problem is not trivial but significant, since this is never solved without Axiom 1 (measurement) and Axiom 2 (causality) (i.e., the answers to the problems "What is measurement?" and "What is causality?").

\section{Conclusion: To Do Science Is to Describe Phenomena by Quantum Language}

\subsection{Summary of Comparison between Logic (in Ordinary Language), Statistics and Quantum Language}

"What is science?" is the main question of philosophy of science. There may be the following three answers:

$\left(\#_{1}\right)$ Science is to describe phenomena by logic

$\left(\#_{2}\right)$ Science is to describe phenomena by statistics

$\left(\#_{3}\right)$ Science is to describe phenomena by quantum language

In this paper, we asserted that $\left(\#_{3}\right)$, rather than $\left(\#_{1}\right)$ and $\left.\left(\#_{2}\right)\right]$, more essential. In what follow, again let us examine this:

[( $\left.\#_{1}\right):$ Logic]: Some may say "Science is to describe phenomena by logic", which may be due to the logical positivism (or, the tradition of Aristotle's syllogism). However, as seen in Sections 3-6, Hempel's raven paradox, Hume's problem of induction, Goodman's grue paradox, Peirce's abduction and flagpole problem are related to the concept of measurement (=inference), and thus, these problems cannot be adequately handled by logic alone. Thus, we think that logic is the language of mathematics, and not the language of science. Mathematical logic (i.e., the language of mathematics) should not be confused with usual logic. As seen throughout this paper, we believe that the representation using "logic" is rough in most cases. So-called logic plays an essential role in everyday conversation (e.g., trial, business negotiations, politics, romance, etc.). On the other hand, science requires quantitative discussion, and thus, science may choose statistics (or, quantum language) rather than logic. It should be noted that

[( $\left.\#_{2}\right)$ : Statistics; the classical mechanical world view]: Statistics are used everywhere in science, and thus, statistics may be the principle of science. Therefore some may say "Science is to describe phenomena in the classical mechanical worldview ( $\approx$ statistics $\approx$ dynamical system theory)". This answer may be somewhat better as follows.

$\left(\mathrm{X}_{1}\right)$ economics is to describe economical phenomena by statistics (it is usual to regard economics as the application of dynamical system theory ( $\approx$ statistics))

$\left(\mathrm{X}_{2}\right)$ psychology is to describe psychological phenomena by statistics

$\left(\mathrm{X}_{3}\right)$ biology is to describe biological phenomena by statistics

$\left(\mathrm{X}_{4}\right)$ medicine is to describe medical phenomena by statistics (i.e., medical sta- 
tistics)

Also, since dynamical system theory is considered as a kind of mathematical generalization of Newtonian mechanics, we may be allowed to say:

$\left(\mathrm{X}_{5}\right)$ Newtonian mechanics is to describe classical mechanical phenomena by statistics (=dynamical system theory). Also, it is clear that dynamical system theory plays a central role in engineering.

though Newtonian mechanics is physics, and thus, it belongs to the realistic worldview in Figure 1.

However, statistics ( $\approx$ dynamical system theory (cf. Remark 14$)$ ) is too mathematical. Hence, "Science is to describe phenomena in the classical mechanical worldview ( $\approx$ statistics $\approx$ dynamical system theory)" is almost the same as "Science is to describe phenomena using the mathematical theories of probability and differential equation". And thus, the framework of the classical mechanical worldview is ambiguous. Since statistics ( $\approx$ dynamical system theory) does not have clear axioms, we think that it is a little unreasonable to say that statistics is the language of science.

For example, we don't know how to attack Hempel's raven problem $\left(\mathrm{K}_{3}\right)$ and $\left(\mathrm{K}_{4}\right)$ from the statistical point of view, since statistics does not have the concept of measurement. As seen below, the relationship between science and statistics is revealed by quantum language ( $c f$. (9) in Figure 1).

[ $\left(\#_{3}\right)$ : Quantum language; the linguistic quantum mechanical worldview]: We choose quantum language (i.e., the linguistic quantum mechanical worldview, or the probabilistic interpretation of science), and we assert "Science is to describe phenomena by quantum language". That is, in a similar sense of $\left(\mathrm{X}_{1}\right)-\left(\mathrm{X}_{5}\right)$, we say that

$\left(\mathrm{Y}_{1}\right)$ economics is to describe economical phenomena by quantum language

$\left(\mathrm{Y}_{2}\right)$ psychology is to describe psychological phenomena by quantum language (cf. Chapter 18 in ref [18])

$\left(\mathrm{Y}_{3}\right)$ biology is to describe biological phenomena by quantum language

$\left(\mathrm{Y}_{4}\right)$ medicine is to describe medical phenomena by quantum language

$\left(\mathrm{Y}_{5}\right)$ Newtonian mechanics is to describe classical mechanical phenomena by quantum language (in the same meaning as the $\left(\mathrm{X}_{5}\right)$, also recall the history: (2) $\rightarrow$ (7) $\rightarrow$ (10) in Figure 1). Also, it is clear that classical system theory (=dynamical system theory, $\left.c f .\left(\mathrm{B}_{2}^{\prime}\right)\right)$ plays a central role in engineering.

$\left(\mathrm{Y}_{6}\right)$ statistical mechanics is to describe statistical mechanical phenomena by quantum language (cf. ref. [12])

$\left(\mathrm{Y}_{7}\right)$ quantum mechanics (i.e., quantum information theory) is to describe quantum mechanical phenomena by quantum language $\left(c f .\left(\mathrm{B}_{1}\right)\right)$.

$\left(\mathrm{Y}_{8}\right)$ As mentioned in the (iv) and (v) of Remark 7, a lot of paradoxes (e.g., Bertrand's paradox, McTaggart's paradox, Zeno's paradox, Monty Hall problem, etc. (cf. refs. [11] [17] [18])) are clarified in quantum language.

$\left(\mathrm{Y}_{9}\right)$ As shown in Sections 3-6, fundamental problems in philosophy of science (i.e., Hempel's raven paradox, Hume's problem of induction, Peirce's abduction, 
the flagpole problem, etc.) are easily solved in the linguistic quantum mechanical worldview. Therefore, quantum language guarantees that these problems are scientific. On the other hand, Goodman's grue paradox $\left(\mathrm{R}_{1}\right)$ cannot be described by quantum language. Thus, it is not scientific. Also, it should be noted that these results are consequences of Axiom 1 [measurement].

etc. Quantum language has the advantage of having the concept of "measurement". And thus, as seen in this paper, "logic" can be paraphrased in detail in terms of measurement, and thus, precise expression is obtained.

Remark 26. [Can logic and statistics be regarded as kinds of worldviews?] Logic and statistics has respectively various aspects. However, when we say roughly, logic is the language of mathematics, and statistics is a quite useful mathematical theory. If so, how can we regard logic and statistics as kinds of worldviews? In this paper, we see that logic and statistics respectively has aspects such as simplified forms of quantum language. That is,

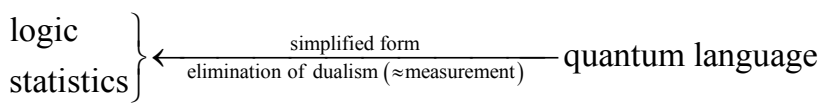

(e.g. see $\left(\mathrm{B}_{3}\right)$ in Figure 1 and the formulas (6), (11)). If so, logic and statistics are scientific as simplified forms of quantum language. Thus, we conclude that so-called logic (i.e., non-mathematical logic) is essential in usual conversation (e.g., argument in a trial, etc.) and not in science.

\subsection{Summing Up; Quantum Language Is the Language of Science}

In this paper, we clarify the following unsolved problems in the linguistic quantum mechanical worldview:

- Hempel's raven paradox in Section 3, Hume's problem of induction in Section 4.1, Goodman's grue paradox in Section 4.2, Peirce's abduction in Section 5, the flagpole problem in Section 6

That is, we sum up as follows:

$\left(\mathrm{Z}_{1}\right)$ [Hempel's raven paradox in Section 3]: Hempel's raven problem (i.e., $\left(\mathrm{K}_{3}\right)$ and $\left.\left(\mathrm{K}_{4}\right)\right)$ is related to measurement and not logic. Thus it is not solved if the concept of measurement is not clarified. Therefore, it is easily solved in the linguistic quantum mechanical worldview since it includes Axiom 1 [measurement].

$\left(\mathrm{Z}_{2}\right)$ [Hume's problem of induction in Section 4.1]: In the linguistic quantum mechanical worldview, Hume's problem of induction (=Theorem 20 [Inductive reasoning]) is essentially the same as the law of large numbers (which is the most basic theorem in science). It may be reasonable. That is because Hume's problem of induction should be the most basic claim in science if there is the answer to this problem.

$\left(Z_{3}\right)$ [Goodman's grue paradox in Section 4.2]: We believe that if Hume's problem of induction is solved, Goodman's grue paradox is immediately solved as its corollary. In fact, Goodman's grue paradox can be easily solved as the corollary of Theorem 20 [Inductive reasoning] in the linguistic quantum mechan- 
ical worldview. Thus, Goodman's grue paradox is due to the lack of the understanding of Hume's problem of induction.

$\left(Z_{4}\right)$ [Peirce's abduction in Section 5]: As shown in the formula (11), Peirce's abduction is characterized as the simplified form of Fisher's maximum likelihood method in quantum language.

$\left(Z_{5}\right)$ [The flagpole problem in Section 6]: The confusion concerning the flagpole problem is due to relying only on "logic", and not using quantum language.

Hence we conclude that the reason that these problems are not yet clarified depends on lack of the worldview with the concept of measurement in philosophy of science. Again we emphasize the importance of worldview in science. That is because, if we do not have the worldview, we do not know what to rely on to proceed with the discussion. Therefore, it is no exaggeration to say that there is no science without a scientific worldview.

As mentioned in Remark 1, quantum language does not cover all sciences. However, we consider that familiar sciences are described by quantum language. And further, we believe that quantum language (i.e., the probabilistic interpretation of science) plays a central role in almost familiar sciences. That is, we believe that quantum language gives the mathematical foundations to science.

However, the answer to "What is science?" may not necessarily be determined uniquely. Although there are several aspects of philosophy of science, we believe that it is the central theme of the philosophy of science to find a mathematical structure that is common to almost all sciences. If so, we feel like knowing other interpretations (i.e., other scientific worldview) besides ours (i.e., the probabilistic interpretation of science). Thus, we hope that various mathematical foundations (e.g., category theoretical approach, modal logic approach, etc.) of scientific philosophy will be proposed. And we expect that fundamental problems such as raven problem, problem of induction, grue paradox, etc. will be investigated in these interpretations. And we hope that philosophy of science will progress with such competition.

We hope that our proposal will be examined from various points of view ${ }^{1}$.

\section{Acknowledgements}

The author wishes to acknowledge Prof. K. Kodaka in Ryukyu University for valuable suggestions.

\section{Conflicts of Interest}

The author declares no conflicts of interest regarding the publication of this paper.

\section{References}

[1] Hempel, C.G. (1945) Studies in the Logic of Confirmation (I). Mind, 54, 1-26. https://doi.org/10.1093/mind/LIV.213.1

${ }^{1}$ For the further information of quantum language, see my home page: http://www.math.keio.ac.jp/ ishikawa/indexe.html. 
[2] Hempel, C.G. (1965) Aspects of Scientific Explanations and Other Essays in the Philosophy of Science. Free Press, New York.

[3] Ishikawa, S. (1991) Uncertainty Relation in Simultaneous Measurements for Arbitrary Observables. Reports on Mathematical Physics, 9, 257-273. https://doi.org/10.1016/0034-4877(91)90046-P

[4] Ishikawa, S. (1997) Fuzzy Inferences by Algebraic Method. Fuzzy Sets and Systems, 87, 181-200. https://doi.org/10.1016/S0165-0114(96)00035-8 http://www.sciencedirect.com/science/article/pii/S0165011496000358

[5] Ishikawa, S. (2000) Statistics in Measurements. Fuzzy Sets and Systems, 116, 141-154. https://doi.org/10.1016/S0165-0114(98)00280-2 http://www.sciencedirect.com/science/article/pii/S0165011498002802

[6] Ishikawa, S. (2006) Mathematical Foundations of Measurement Theory. Keio University Press Inc., Yokohama, 335 p. http://www.keio-up.co.jp/kup/mfomt

[7] Ishikawa, S. (2011) A New Interpretation of Quantum Mechanics. Journal of Quantum Information Science, 1, 35-42. https://doi.org/10.4236/jqis.2011.12005 http://www.scirp.org/journal/PaperInformation.aspx?paperID $=7610$

[8] Ishikawa, S. (2012) Quantum Mechanics and the Philosophy of Language: Reconsideration of Traditional Philosophies. Journal of Quantum Information Science, 2, 2-9. http://www.scirp.org/journal/PaperInformation.aspx?paperID=18194 https://doi.org/10.4236/jqis.2012.21002

[9] Ishikawa, S. (2012) The Linguistic Interpretation of Quantum Mechanics. http://arxiv.org/abs/1204.3892

[10] Ishikawa, S. (2012) A Measurement Theoretical Foundation of Statistics. Applied Mathematics, 3, 283-292. https://doi.org/10.4236/am.2012.33044 http://www.scirp.org/journal/PaperInformation.aspx?paperID=18109

[11] Ishikawa, S. (2012) Monty Hall Problem and the Principle of Equal Probability in Measurement Theory. Applied Mathematics, 3, 788-794. https://doi.org/10.4236/am.2012.37117 http://www.scirp.org/journal/PaperInformation.aspx?PaperID=19884

[12] Ishikawa, S. (2012) Ergodic Hypothesis and Equilibrium Statistical Mechanics in the Quantum Mechanical World View. World Journal of Mechanics, 2, 125-130. https://doi.org/10.4236/wjm.2012.22014 http://www.scirp.org/journal/PaperInformation.aspx?PaperID=18861\#.VKevmius Wap

[13] Ishikawa, S. (2015) Linguistic Interpretation of Quantum Mechanics; Projection Postulate. Journal of Quantum Information Science, 5, 150-155. https://doi.org/10.4236/jqis.2015.54017 http://www.scirp.org/Journal/PaperInformation.aspx?PaperID=62464

[14] Ishikawa, S. (2017) History of Western Philosophy from the Quantum Theoretical Point of View. Research Report, Department of Mathematics, Keio University, Yokohama, Ver. 2, KSTS-RR-17/004, 141 p. http://www.math.keio.ac.jp/academic/research_pdf/report/2017/17004.pdf

[15] Ishikawa, S. (2017) A Final Solution to Mind-Body Problem by Quantum Language. Journal of Quantum Information Science, 7, 48-56. https://doi.org/10.4236/jqis.2017.72005 http://www.scirp.org/Journal/PaperInformation.aspx?PaperID=76391

[16] Ishikawa, S. (2017) Bell's Inequality Should Be Reconsidered in Quantum Language. Journal of Quantum Information Science, 7, 140-154. https://doi.org/10.4236/jqis.2017.74011 
http://www.scirp.org/Journal/PaperInformation.aspx?PaperID=80813

[17] Ishikawa, S. (2018) Leibniz-Clarke Correspondence, Brain in a Vat, Five-Minute Hypothesis, McTaggart's Paradox, etc. Are Clarified in Quantum Language. Open Journal of Philosophy, 8, 466-480. https://doi.org/10.4236/ojpp.2018.85032 https://www.scirp.org/Journal/PaperInformation.aspx?PaperID=87862

[18] Ishikawa, S. (2018) Linguistic Copenhagen Interpretation of Quantum Mechanics; Quantum Language. Research Report, Dept. Math., Keio University, Yokohama, Ver. 4, KSTS/RR-18/002, 449 p. http://www.math.keio.ac.jp/academic/research_pdf/report/2018/18002.pdf

[19] Putnam, H. (1981) Reason, Truth and History. Cambridge University Press, Cambridge. https://doi.org/10.1017/CBO9780511625398

[20] von Neumann, J. (1932) Mathematical Foundations of Quantum Mechanics. Springer Verlag, Berlin.

[21] Prugovečki, E. (1982) Quantum Mechanics in Hilbert Space. Academic Press, New York.

[22] Sakai, S. (1971) $C$-Algebras and $W^{*}$-Algebras, Ergebnisse der Mathematik und ihrer Grenzgebiete (Band 60). Springer-Verlag, Berlin, Heidelberg, New York.

[23] Yosida, K. (1980) Functional Analysis. Sixth Edition, Springer-Verlag, Berlin.

[24] Davies, E.B. (1976) Quantum Theory of Open Systems. Academic Press, Cambridge.

[25] Howard, D. (2004) Who Invented the "Copenhagen Interpretation"? A Study in Mythology. Philosophy of Science, 71, 669-682. https://doi.org/10.1086/425941

[26] Kolmogorov, A. (1960) Foundations of the Theory of Probability (Translation). Second Edition, Chelsea Pub. Co., New York.

[27] Popper, K.R. (1959) The Logic of Scientific Discover. Physics Today, 12, 53. https://doi.org/10.1063/1.3060577

[28] Goodman, N. (1983) Fact, Fiction, and Forecast. Harvard University Press, Cambridge.

[29] Peirce, C.S. (1958) Collected Papers of Charles Sanders Pierce. Harvard University Press, Cambridge. 